\title{
Aux origines de la cité de cheminots de Lille-La Délivrance (1921-1926)
}

\section{Arnaud Gaboriau}

\section{OpenEdition}

\section{Journals}

Édition électronique

URL : https://journals.openedition.org/rhcf/692

DOI : $10.4000 /$ rhcf.692

\section{Éditeur}

Rails \& histoire

\section{Édition imprimée}

Date de publication : 1 mai 2004

Pagination : 101-138

ISSN : 0996-9403

\section{Référence électronique}

Arnaud Gaboriau, «Aux origines de la cité de cheminots de Lille-La Délivrance (1921-1926) », Revue

d'histoire des chemins de fer [En ligne], 31 | 2004, mis en ligne le 08 avril 2011, consulté le 22 avril 2022. URL : http://journals.openedition.org/rhcf/692 ; DOI : https://doi.org/10.4000/rhcf.692

Ce document a été généré automatiquement le 22 avril 2022.

Tous droits réservés 


\title{
Aux origines de la cité de cheminots de Lille-La Délivrance (1921-1926)
}

\author{
Arnaud Gaboriau
}

Décembre 1920 : les habitants de la paisible commune de Lomme, petite ville de la banlieue lilloise, voient plus de cent soixante-dix d'hectares de champs humides se transformer en un immense chantier; en l'espace de quelques mois surgit de nulle part un important complexe ferroviaire baptisé Lille-La Délivrance, comprenant une gare de triage ainsi qu'une cité-jardin destinée à loger le personnel nécessaire à l'exploitation du site. Juillet 1926: le ministère des Travaux publics et des transports approuve, par décision à titre de régularisation comme suite et complément des travaux déclarés d'utilité publique pour la construction de la ligne d'Haubourdin à Saint-André, le projet d'établissement de la cité cheminote de Lille-La Délivrance.

Durant ces six années apparaît donc à proximité immédiate d'un des plus grands centres ferroviaires de la région lilloise une nouvelle agglomération dotée de toutes les "modernités ", qui forme une entité géographique de la dimension d'une petite ville : près de soixante-dix hectares, plus de huit cent trente logements, trois mille trois cents habitants. L'étude de la naissance d'une telle réalisation présente bien des intérêts. Tout d'abord elle procède d'une autre logique que celle qui anime les agglomérations voisines du Bourg, du Marais et de Canteleu. Créée ex nihilo au lendemain de la Première Guerre mondiale, elle n'est pas le fruit d'une lente et longue évolution mais doit son existence à la volonté d'une société, la Compagnie du chemin de fer du Nord, et d'un homme, l'ingénieur Raoul Dautry. Les conditions particulières de sa naissance lui confèrent ainsi une identité propre et en font un véritable monde à part. Par ailleurs, elle bénéficie d'une architecture de qualité, son esthétique, soignée, rompant avec celle des cités ouvrières traditionnelles. 


\section{La genèse de la gare et de la cité de Lille-La Délivrance}

\section{La naissance de la gare de Lille-La Délivrance}

3 Le bilan de la Grande Guerre se révèle pour la Compagnie du Nord extrêmement lourd. $\mathrm{Au}$ lendemain de l'armistice, la quasi-totalité du réseau est détruite. Les armées allemandes ont pris soin, durant leur retraite, de mettre hors d'usage les voies et installations ferroviaires. En témoigne cet extrait d'un rapport présenté par le conseil d'administration de la compagnie : «le réseau se trouve, sur 2123 kilomètres, sans un seul pont ou tunnel existant, sans un seul dépôt de locomotives, sans une seule gare, grande ou moyenne, qui n'aient pas été à peu près complètement détruits. Les voies elles-mêmes ont été, sur des kilomètres, détruites, soit par une charrue spéciale imaginée par les Allemands et traînée par une locomotive arrachant, derrière elle, les rails des traverses, soit par explosifs mettant les rails hors de service en faisant sauter un joint sur deux ${ }^{1}$.» Quant à la partie du réseau qui a pu échapper à ces mesures de destruction systématique, l'intensification de son exploitation durant le conflit lui a fait subir une usure précoce.

4 En un laps de temps extrêmement court, la compagnie parvient à rétablir les voies principales et à remettre en état les gares les plus importantes : «la reconstitution du réseau de la Compagnie du Nord a fait au cours de l'exercice 1919 de très grands progrès. [...] Depuis lors, l'exploitation a pu être complètement reprise. Après les rails, les ponts et les tunnels ont été à peu près entièrement rétablis. [...] C'est un spectacle aussi réconfortant qu'inattendu de voir se dérouler ces lignes déjà rétablies dans leur belle tenue d'autrefois au milieu de la zone dévastée qu'elles traversent ${ }^{2}$.» Ainsi, dès avril 1919, la circulation est rétablie sur l'ensemble des lignes du réseau. Cependant cette œuvre de reconstruction remarquable par sa rapidité ne suffit pas: les installations, à peine réouvertes, sont déjà saturées. Leur décongestion nécessite la réalisation d'une nouvelle infrastructure de gares, dépôts et triages plus vastes, adaptés aux nouveaux besoins et capables de faire face aux pointes de trafic les plus fortes. C'est dans ce contexte de modernisation du réseau que s'inscrit le projet visant à édifier à l'ouest de l'agglomération lilloise une nouvelle grande gare de triage.

5 Ce projet n'est pas nouveau. Dès 1911 le centre de Fives, par lequel passe tout le trafic marchandises et voyageurs, se révèle insuffisant pour assurer le triage des wagons et la formation des trains : «ce trafic était devenu si chargé qu'on ne pouvait l'assurer que par des prodiges d'habileté et grâce à un entraînement remarquable du personnel ${ }^{3}$. » La compagnie doit créer un triage d'appoint à Rougebarre, aux confins des communes de Marcq-en-Barœul, Mons-en-Barœul et Wasquehal, sur le raccordement reliant la ligne de Dunkerque à celle de Tourcoing, tandis que la gare de La Madeleine, mieux outillée, apporte une aide complémentaire ${ }^{4}$. De telles méthodes ne sont évidemment pas satisfaisantes, le fractionnement du travail et les multiples sujétions qui en découlent présentant de trop nombreux inconvénients. Est alors envisagée la réalisation d'une grande gare de triage qui absorberait et centraliserait à elle seule toutes les opérations de triage et de formation des trains. Le projet est cependant à peine ébauché lorsque la guerre éclate.

6 Le 2 mai 1919 les services de la compagnie présentent un ensemble de croquisprogrammes en vue de la détermination du terrain devant accueillir la future gare de triage de Lille-La Délivrance : leur choix se porte sur une centaine d'hectares presque 
inhabitée, située pour l'essentiel sur le territoire de la commune de Lomme ${ }^{5}$, entre les hameaux du Bourg et du Marais. Le 25 août, une décision ministérielle approuve les propositions de la compagnie. L'emplacement retenu offre «l'avantage de border, sur une de ses limites, la voie circulaire qui entoure la ville de Lille et dessert les gares de débord de la ceinture de Lille correspondant soit à des quartiers excentrés de la ville, soit à des communes suburbaines: Fives, Lille-Saint-Sauveur, Lille-Sud, Loos, Haubourdin, Sequedin, Lomme, Lambersart, Saint-André et La Madeleine $»^{6}$. De plus, ces vastes étendues de terrains humides (l'eau s'y trouve à moins d'un mètre de profondeur), sur lesquelles ne sont implantées que quelques fermes, n'ont que peu de valeur et leur acquisition, dégagée des contraintes foncières urbaines, peut être relativement peu coûteuse.

$7 \quad$ Le choix du site ne s'explique pas uniquement par des considérations d'ordre technique et financier. Le projet ferroviaire comprend, outre la gare proprement dite, l'édification d'une cité destinée à assurer le logement des cheminots. Or les administrateurs de la compagnie souhaitent que les cités soient construites loin des villes et des faubourgs ouvriers, foyers de l'agitation révolutionnaire et contestataire en cette terre historique du syndicalisme et du socialisme, afin d'éviter toute contagion ${ }^{7}$. De plus, comme le souligne Pierre Outteryck, ils n'hésitent pas, lorsqu'ils en ont la possibilité, à les implanter sur le territoire de communes réputées pour leur électorat modéré8. Force est de reconnaitre que le site retenu répond à ces deux préoccupations, éloigné des quartiers populaires lillois, implanté sur une terre marécageuse non loin du quartier du Bourg, jugé majoritairement conservateur.

8 Pourquoi avoir donné à la gare (et par extension à la cité de cheminots) le nom de «Lille-La Délivrance »? Jules Brenne fournit une réponse à cette question : « On a fait quant à ce nom des suppositions diverses. [...] Le vrai récit de ce baptême, maintenant presque oublié, a été fait par maître Decroix, alors qu'il était vice-président de la Commission historique du Nord. Avocat, bâtonnier de l'ordre, il participait en 1919, comme conseiller juridique à la réunion à Paris au cours de laquelle le nom devait être choisi. Monsieur Javary la présidait. Ingénieur en chef de la Compagnie des chemins de fer du Nord, c'était un homme fort important. Les techniciens et juristes présents avaient proposé divers noms. L'ingénieur en chef s'est tout d'un coup, prononcé souverainement: "Messieurs, la gare nouvelle sera Lille-La Délivrance". Lille et sa région avaient été occupées quatre ans par l'ennemi. Ce qui s'est appelé Libération en 1944 se disait Délivrance en 1918. L'événement était tout récent et le nom avait une résonance joyeuse ${ }^{9}$.»

\section{La question du logement des cheminots et le principe des cités}

9 Cette initiative en matière de logement ne constitue pas un cas unique, mais s'inscrit pleinement dans le cadre d'une grande opération immobilière menée par la Compagnie du Nord au lendemain de la guerre.

La paix revenue, le Nord doit faire face à une grave crise du logement : « la guerre a brusquement arrêté les constructions qui, chaque année, augmentaient de plusieurs milliers de nouvelles maisons ouvrières le patrimoine immobilier du département $\mathrm{du}$ Nord. Elle a en outre causé à ce patrimoine d'immenses dommages matériels. Elle a notamment détruits nombre d'établissements industriels dont beaucoup ne sont pas reconstruits sur place. [...] Les usines nouvelles, recherchant de vastes terrains à bon 
marché, des communications faciles par eau et par fer, se sont créées fort loin parfois de celles qu'elles remplacent, entraînant ainsi le déplacement d'une main-d'œuvre dont la première et fort naturelle exigence est de posséder un logement à proximité de son travail. En attendant qu'il puisse être satisfait à cette nécessité, les usines se voient encore contraintes de recruter leur personnel dans un rayon beaucoup trop étendu et de l'envoyer prendre, parfois jusqu'à trente kilomètres, en camions automobiles. [...] Toutes ces raisons réunies ont donné à la crise du logement dans le Nord une intensité particulière $[. ..] »^{10}$ : elle n'épargne pas les cheminots parmi lesquels figurent bon nombre de sinistrés ou de mal logés. Les administrateurs de la compagnie décrivent en ces termes la situation: «la question du logement des familles de nos agents dans les régions dévastées se pose, à l'heure actuelle avec un caractère d'urgence tout à fait

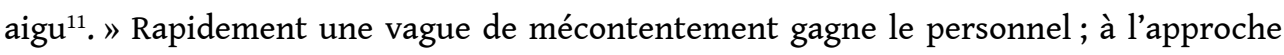
du Premier mai 1919 le ton se durcit. Le syndicat Paris-Nord de la Fédération nationale des travailleurs des chemins de fer de France et des colonies réclame l'augmentation des salaires et la retraite à 50 ans tandis que l'idée de la nationalisation des chemins de fer est remise sur le tapis. Alors que de nombreuses rumeurs de grève circulent, le syndicat de Paris-Nord réclame l'établissement définitif des soviets sur le réseau.

11 C'est aussi pour contribuer à désamorcer la révolte qui gronde et éviter que ne se reproduise une grande grève générale comparable à celle de 1910, dont le souvenir reste présent dans l'esprit des membres dirigeants de la compagnie, qu'est confiée à l'ingénieur Raoul Dautry la mission de résoudre le délicat problème du logement des agents. Il fait de suite répartir dans l'ensemble des districts des maisons provisoires mais ces mesures prises dans l'urgence, si elles permettent de parer au plus pressé, ne peuvent cependant constituer une réponse adaptée à long terme, d'autant plus que la modernisation du réseau ainsi que l'application de la loi du 23 avril 1919 sur la journée de huit heures nécessitent le recrutement de milliers de nouveaux agents. Pour assurer le logement de ce personnel de plus en plus nombreux, est finalement décidée, sur la proposition de Raoul Dautry, l'édification à proximité des installations ferroviaires de cités-jardins.

Entre 1919 et 1924 une trentaine de cités-jardins, dont 25 de plus de 100 logements, sont créées dans le cadre d'une opération immobilière sans précédent concernant plus de 11200 habitations. Lille-La Délivrance, avec ses 835 logements, constitue la citéjardin la plus importante après celle de Tergnier (1 112 logements) (tabl. 1).

Tableau 1. Cité-jardins de la Compagnie du Nord de plus de 100 logements au 31 décembre 1924.

\begin{tabular}{|l|l|}
\hline Cité-jardins de plus de 1000 logements : & $\begin{array}{l}\text { Cités-jardins comptant de } 101 \quad \text { à } 200 \\
\text { logements : }\end{array}$ \\
\hline , Tergnier & , Busigny \\
\hline $\begin{array}{l}\text { Cités-jardins comptant entre } 601 \text { et } 850 \\
\text { logements: }\end{array}$ & , Douai \\
\hline , Lille-La Délivrance & , Montdidier \\
\hline , Lens & , Cambrai \\
\hline , Dunkerque & \\
\hline
\end{tabular}




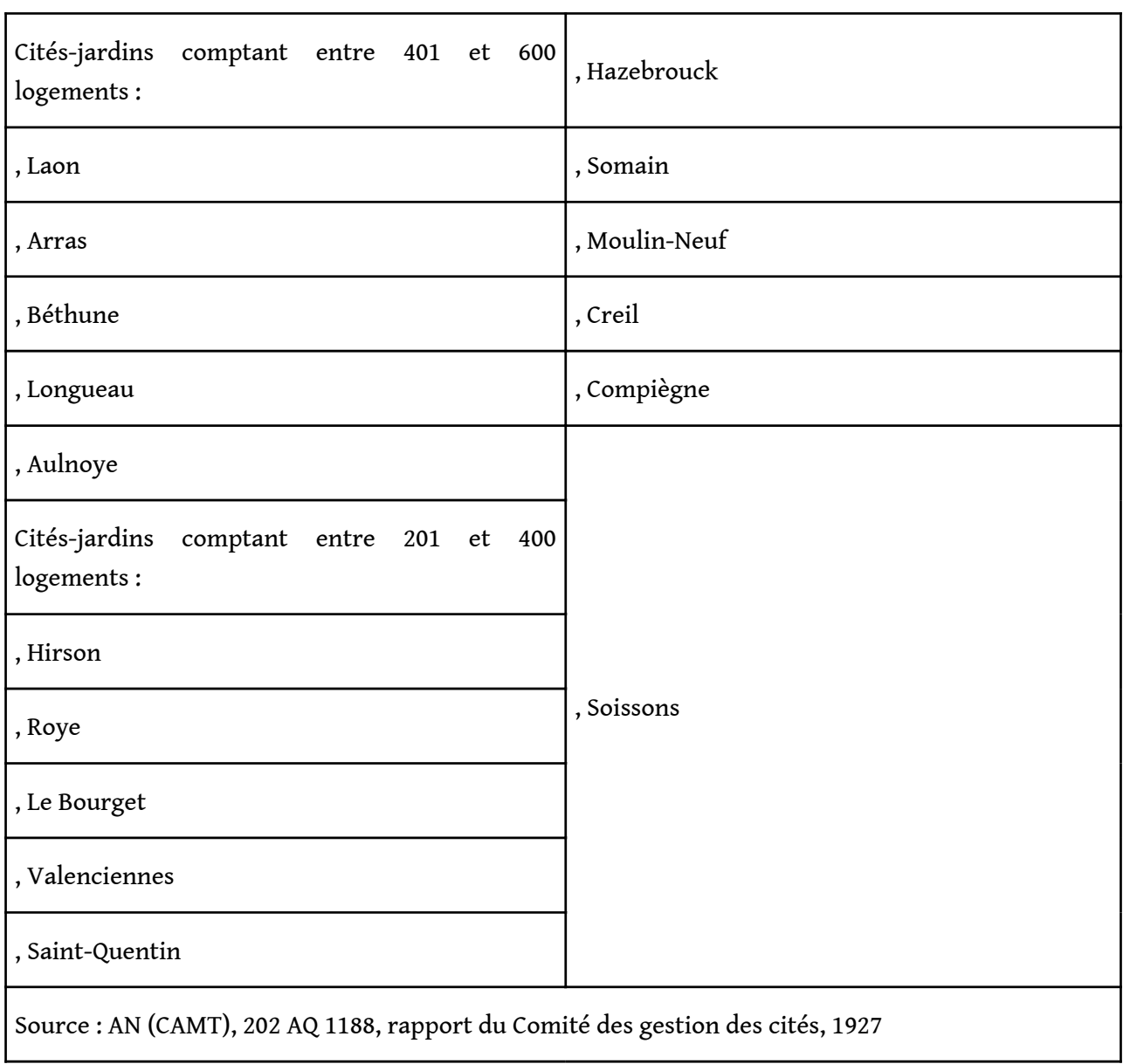

Il est à noter que les premières cités de cheminots réalisées par la Compagnie du Nord sont antérieures à la Première Guerre mondiale. Cette dernière entreprend en effet dès 1883 l'édification de quelques cités sur certains points du réseau où les ressources en habitations s'avèrent limitées : sont ainsi construites dans un premier temps les cités d'Aulnoye, Ermont, Le Bourget, Lens, Somain et Coudekerque puis, quelques décennies plus tard, celles de Longueau, Guise et Bully-Grenay. Au final, ce sont donc neuf cités qui voient le jour avant 1914.

13 Les cités sont considérées par la compagnie comme un moyen efficace d'attirer et de fixer la main-d'œuvre et, à ce titre, comme un véritable « investissement productif ». Dans une région comme celle du Nord, très industrialisée, les entreprises se livrent dès la fin du dix-neuvième siècle une concurrence acharnée pour faire venir les ouvriers qualifiés. Cette situation se fait encore plus vivement sentir au lendemain de la Première Guerre, lors de cette période de reconstruction durant laquelle la France, et particulièrement les territoires septentrionaux et orientaux qui ont subi de terribles dommages au cours du conflit, se transforment en un immense chantier. Le plein emploi et la pénurie de main-d'œuvre offrent alors aux ouvriers la possibilité de changer aisément d'employeur en fonction d'appâts salariaux ou d'avantages particuliers. Dans un tel contexte, le fait «d'offrir » un logement confortable implanté dans une cité dotée d'équipements modernes constitue un argument pour attirer de nouveaux agents ainsi qu'un puissant moyen de stabilisation de ces derniers.

Les cités permettent en outre d'exercer un contrôle étroit sur l'ensemble de la communauté cheminote. Les responsables du réseau du Nord entendent contrer l'influence grandissante des mouvements syndicaux et politiques de gauche, faire barrage à la contagion révolutionnaire, en créant « une culture d'entreprise dont le 
cœur serait l'intégration des travailleurs à une stratégie capitaliste, à l'élaboration de laquelle ils ne participent pas $»^{12}$. Tout est donc mis en œuvre pour que les agents et leur famille qui vivent au sein des cités se sentent membres d'une même corporation: ils y trouvent tout ce dont ils ont besoin (écoles, magasins, bains-douches...), y vivent au même rythme, assimilent les mêmes rapports de convivialité, d'entraide, partagent les mêmes joies et les mêmes soucis. Vivant entièrement à l'heure de la compagnie, les cités facilitent la tâche des ingénieurs, des chefs d'équipes, des gardiens et mouchards en tout genre. Elles permettent d'obtenir des cheminots une entière disponibilité. Logés à proximité de leur lieu de travail, réunis dans les mêmes lieux, ils peuvent être appelés à n'importe quelle heure du jour et de la nuit ; c'est une forme d'astreinte qui ne dit pas son nom. De plus, d'une cité à l'autre, les repères ne changent pas, ce qui contribue à favoriser la mobilité des agents et à rendre les mutations moins stressantes: les femmes des travailleurs acceptent plus volontiers de quitter leur logement et l'environnement dans lequel elles vivent quand elles savent qu'elles vont retrouver des conditions de vie identiques.

Le discours officiel de la Compagnie du Nord ne reflète évidemment pas toutes ces arrière-pensées. Dans les journaux et les diverses brochures illustrées distribuées aux actionnaires et aux parlementaires, l'action de la direction en matière de logement, quelle qu'en soit l'inspiration, est présentée sous l'aspect "social», et rejoint les courants de pensée contemporains. La publicité, dont Raoul Dautry a le génie, n'est pas absente : en témoigne cet extrait d'un article paru dans L'Écho de Paris du 18 août 1924 et intitulé «Réalisations sociales. Les cités-jardins sur le Nord»: «Plus de 12000 maisons [...] aussi confortables que convenablement appropriées aux familles pour lesquelles elles sont destinées, sont aujourd'hui à la disposition du personnel moyennant un loyer modéré, qui rémunère faiblement à $1 \%$ les capitaux engagés. Cet intérêt restreint montre que par cette vaste entreprise la compagnie n'a pas eu l'intention de se lancer dans une affaire rémunératrice ; son désintéressement s'affirme davantage encore, si l'on envisage les conditions dans lesquelles elle a entendu assurer la liberté de jouissance des occupants; elles sont bien faites pour montrer à quelle point le patronat sait, quand il le faut, s'effacer pour satisfaire aux goûts, aux désirs, aux besoins de la classe ouvrière. [...] Il n'est donc plus permis [...] de parler ici d'ingérence du patron dans la vie privée de son personnel; nous sommes loin de cet étroit paternalisme d'antan, qu'on a jadis tant reproché aux patrons qui se mêlaient d'œuvres sociales ${ }^{13}$. »

\section{Une cité-jardin}

\section{Une cité « rationalisée »}

Il est impossible d'aborder l'histoire de la cité de Lille-La Délivrance sans évoquer la personnalité de son véritable concepteur, l'ingénieur Raoul Dautry ${ }^{14}$.

Polytechnicien, Raoul Dautry débute sa carrière au sein de la Compagnie du chemin de fer du Nord en octobre 1903, en tant que simple attaché au service de la Voie. Homme d'initiative et de responsabilité, il gravit avec célérité échelons et grades : en janvier 1906 il devient chef de section, en juillet 1908 inspecteur attaché au service des bâtiments, en décembre 1911 sous-ingénieur en chef adjoint au service des bâtiments. Enthousiaste, assoiffé de connaissances, il s'intègre dans le monde du rail en un laps de 
temps record. Très rapidement, il échafaude une véritable doctrine sur le rôle que doit remplir l'ingénieur au sein de l'entreprise, doctrine relayée par ses nombreuses lectures dans des domaines aussi divers que l'histoire, la philosophie, la politique ou la littérature. Ainsi, à l'instar du capitaine Lyautey qui s'employa à améliorer le sort matériel de ses soldats et à développer leur éducation selon un programme de cours et de conférences, il estime que l'ingénieur doit tout mettre en œuvre pour améliorer les conditions de vie et de travail de ses employés car, écrit son biographe, «clef de voûte de tout progrès, l'action sociale facilite [à terme] la rationalisation des secteurs économiques de production, en bannissant tout conflit dans l'entreprise $»^{15}$. "Son programme social est [donc] d'abord formulé sur les bases d'une réponse technique susceptible de créer une meilleure organisation de la production [...] et de favoriser en même temps la productivité des agents ${ }^{16}$."

Désireux d'obtenir gain de cause et de mettre en application rapidement ses théories, Dautry « bombarde » dans un premier temps, sans véritables résultats, sa hiérarchie de notes et de rapports dans lesquels il s'attache à démontrer la nécessité de construire des économats, des logements sociaux, des cantines et des dispensaires. Finalement, après quelques années d'attente, sa nomination en 1913 à la tête de l'atelier de la voie d'Ermont lui offre la possibilité d'expérimenter sa "doctrine». Chargé durant les hostilités d'y diriger les cheminots employés à la fabrication des équipements ferroviaires nécessaires à la défense nationale, il y met en place une nouvelle organisation du travail. Soucieux d'accroître la production, il transforme ainsi le petit atelier en une véritable zone industrielle. Tandis que sont achetées de nouvelles machines ainsi que des engins de manutention qui facilitent la suppression des « temps morts", est engagé un véritable programme de construction de logements et d'équipements sociaux: dortoirs, réfectoires, cuisines, infirmerie et même jardins ouvriers. Le succès de l'ensemble de l'opération finit par convaincre ses supérieurs du bien fondé de ses méthodes. C'est donc tout naturellement vers lui que se tourne, au lendemain du conflit, la direction de la compagnie pour résoudre la délicate question du logement des cheminots : en octobre 1919, il est nommé à cet effet ingénieur en chef adjoint de l'entretien. "Il théorise [alors] les postulats d'une politique de construction de cités-jardins cheminotes : répondre au problème du logement des agents, c'est-àdire définir les conditions d'une nouvelle société cheminote; de cette dernière découlera un ensemble de bienfaits qui bouleverseront les conditions de production: accroissement de la productivité, de la sécurité et augmentation de la responsabilisation des agents, abolition des conflits capital / travail, enfin création d'une identité cheminote ${ }^{17}$.» Face à la ville qu'il juge «surpeuplée, socialement dangereuse » et à la banlieue qu'il qualifie de « anarchique et affreuse », Dautry entend ainsi établir un nouveau modèle de cité au sein de laquelle "l'agglomération rationalisée et harmonieuse des familles dans des cadres ensoleillés, aérés, organisés, desservis [pourra] seule augmenter le nombre, la qualité et le rendement des individus $»^{18}$.

Lille-La Délivrance est donc conçue sur ce modèle de cité entièrement " rationalisée ", qui vise à « façonner l'ouvrier idéal $»^{19}$.

20 Formant une entité géographique de la dimension d'une ville, d'une superficie de 69 hectares, 27 ares, 64 centiares, son implantation, sa situation, son inscription dans le paysage environnant la désignent comme procédant d'un autre ordre, d'une autre logique que celle qui anime les petites agglomérations voisines. Implantée à l'écart de 
ces dernières, en retrait de la route nationale de Lille à Dunkerque, loin des faubourgs ouvriers lillois, elle apparaît en effet comme un véritable îlot entièrement replié sur lui-même. Espace bien délimité, bordé à l'Est par les voies ferrées de la gare de triage et au Nord par le chemin vicinal ordinaire $n^{\circ} 3$ dit rue Anne-Delavaux, son accès y est de plus verrouillé et contrôlé par un système de places : au Sud la place François-Marcq, à l'Ouest la place Jules-Duhoo, au Nord la place Jules-Delvoye et à l'Est la place LouisTrocmet. Cet isolement est voulu par Dautry qui entend ainsi contrôler étroitement la vie des cheminots et de leur famille, rythmée dès lors par le mouvement et le sifflet des trains qui circulent à proximité. Il est même renforcé d'une "ceinture verte ", censée préserver les habitants de la cité tant des risques extérieurs de pollution idéologique, en cette terre historique de syndicalisme et de socialisme, que de la laideur et du caractère malsain des villes environnantes; en témoigne cet extrait d'une thèse de doctorat portant sur les œuvres sociales de la Compagnie du Nord où il est expliqué que «dans les [...] cités plantées au milieu d'horribles masures, que l'on rencontre si souvent, autour des grands centres, [comme c'est le cas] à Lille et à Valenciennes par exemple, les créateurs [...] ont cherché à les isoler en plantant d'épais rideaux d'arbres, qui semblent actuellement peu de choses, mais qui, dans quelques années, les entoureront d'une ceinture de verdure $»^{20}$.

21 Si Dautry s'attache à faire de la cité un véritable "bastion", imperméable aux influences extérieures qui pourraient se révéler néfastes pour les intérêts de la compagnie, il met aussi tout en œuvre pour obtenir un ensemble urbanistique et architectural agréable, «ne rappelant ni la caserne, ni l'usine, ni le coron $»^{21}$.

22 Le plan de Lille-La Délivrance, "qui représente assez bien une vaste étoile ${ }^{22}$, se caractérise par deux grands axes qui se croisent en leur milieu. Le plus important, formé par les avenues du Beau-Visage et aujourd'hui Roger-Salengro, traverse la cité d'une extrémité à l'autre, reliant la place Louis-Trocmet à la place Jules-Duhoo. Le second, orienté sud-ouest - nord-est, est constitué par l'avenue de la Délivrance et aboutit à la place Jules-Delvoye. Ces deux artères principales, droites, bordées d'arbres d'alignement, servent de support à la création de trois places spacieuses qui apportent une touche de "fantaisie" à ce schéma méthodique et rigoureux: la place de la Victoire, la place Demory et la place Beaulieu. Par ailleurs, de ces dernières et des quatre places périphériques partent en étoile tout un lacis de rues secondaires, «dont les rayonnements ingénieux, les courbes élégantes, les entrecroisements imprévus assurent à l'ensemble une dissymétrie pittoresque $»^{23}$. Qu'il s'agisse donc de dessiner les rues et allées selon un tracé incurvé (ce qui permet d'obtenir des variations de perspectives) ou de couper les boulevards de vastes ronds-points, toutes ces initiatives permettent d'échapper au modèle "des villes modernes, aux voies rectilignes, parallèles et perpendiculaires, construites en Amérique ou en Allemagne à la fin du siècle dernier $»^{24}$.

Parallèlement, l'ensemble réalisé offre l'aspect d'une agglomération vaste et aérée au sein de laquelle «l'élément nature » occupe une place primordiale. L'introduction de ce dernier dans le paysage de la cité revêt des formes multiples et variées. Les sept places, qui ont un rayon de cinquante mètres en moyenne, sont encadrées par des haies d'arbustes tandis que des gazons, des massifs de fleurs les égaient. Parfois des motifs décoratifs, une fontaine ou un vieux puits en ornent le centre ${ }^{25}$; c'est le cas notamment de la place Beaulieu ${ }^{26}$ " au centre [de laquelle] se dresse l'un de ces énormes vases de Sèvres composés par l'architecte P. Patout et qui flanquaient le joli jardin de la 
manufacture à l'Exposition des Arts décoratifs $»^{27}$. Ces squares constituent des lieux de vie publics et de sociabilité intense : ils permettent les jeux de plein $a^{2}{ }^{28}$ et offrent dans certains cas un cadre rêvé pour l'organisation de "fêtes champêtres ». Le "magnifique verger de 23 ares qui existait lors de la fondation de la cité ${ }^{29}$, situé entre les places de la Victoire, Beaulieu et Jules-Delvoye, est quant à lui un véritable "poumon vert ", formant un océan de verdure au milieu duquel baignent, entre autres édifices, la salle des fêtes et de réunion ainsi que l'école maternelle. Non seulement la cadre végétal apporte "fraîcheur et gaieté ", "éloignant le cheminot, sa femme, ses enfants, des plaisirs vulgaires et favorisant un des deux plus nobles plaisirs des sens : celui de la vue $\aleph^{30}$, mais il joue aussi un rôle structurant. Tilleuls, acacias, marronniers bordent en effet les avenues, larges de douze mètres, soulignent les places tandis que des haies, supportées par plus de 70 kilomètres de clôtures en fil de fer, longent les allées et délimitent les jardins : "pour éviter la perspective déplaisante des rues bordées de murs, [...] on a constitué les clôtures des jardins, extérieures ou mitoyennes, par de simples fils de fer, portés par des potelets en béton armé $»^{31}$. Enfin, les jardins individuels permettent une appropriation privée du cadre végétal. D’une superficie de 400 à 500 mètres carrés, ils entourent chacune des maisons construites; ces dernières, qui ne regroupent jamais plus de quatre logements ${ }^{32}$, sont ainsi isolées les unes des autres, disséminées le long des voies et allées, donnant l'image de petits "chalets » dont la « variété de lignes compose sur le fond vert des pelouses et des jardinets [...] un ensemble plein d'harmonie $»^{33}$. L'abandon du principe des immeubles en mitoyen et de l'alignement strict des façades sur la rue au profit d'édifices séparés et d'espaces extérieurs plus ouverts, avec une forte présence de la végétation, contribue largement à expliquer la faible densité de la cité: en 1926, elle compte "seulement" 835 logements et 3228 habitants, soit 12 logements et 47 habitants par hectare ${ }^{34}$.

Cette grande réalisation architecturale est systématiquement présentée par ses concepteurs sous le terme de "cité jardin ». Cette appellation est reprise, par ailleurs, par les auteurs des articles parus dans la presse que nous qualifierons de " professionnelle ", qu'il s'agisse de La Construction moderne, L'Architecture usuelle ou Le Génie civil. L'idée de cité-jardin dérive du livre de l'Anglais Ebenezer Howard (1850-1928) intitulé Tomorrow: a Peaceful Path to Real Reform (1898), dans lequel, en réponse aux problèmes posés par l'industrialisation et la croissance démographique, il préconise la construction d'une série de "garden cities ». " [Unissant] les bienfaits de la ville - la vie sociale, les services publics - avec ceux de la campagne, la verdure, la tranquillité, la salubrité, etc. $»^{35}$, ces nouvelles villes seraient indépendantes les unes des autres, aussi bien économiquement que structurellement. Le succès de cette proposition est immédiat: dès 1903 est inaugurée la cité de Letchworth, première "garden city" et "premier modèle social et architectural $d u x^{e}$ siècle tout en s'inspirant des modèles du logement industriel du XIX ${ }^{e}$ siècle que sont encore Port Sunlight (1888) et Bournville (1895) $»^{36}$. Le mouvement pour les "garden cities» se diffuse rapidement sur le continent. En France, le concept de cité-jardin est introduit par le juriste Georges Benoît-Levy (1880-1971) qui, en 1903, à l'initiative du Musée social, se rend en Angleterre afin de rendre compte de l'expérience de Letchworth. Très impressionné par la nouvelle ville, il publie dès son retour son rapport «La cité-jardin " en forme de manifeste et crée l'Association des cités-jardins de France. Quelques années plus tard, en 1908, est constituée la Section d'hygiène urbaine et rurale du Musée social qui élabore le mouvement composite du projet de cité-jardin : animé par des associations issues de divers milieux socioprofessionnels, ce dernier propose, en 
réponse à la crise du logement, à la croissance monocentrique des métropoles industrielles et à l'extension sans fin des banlieues anarchiques, la construction de cités-jardins au pourtour des agglomérations. La cité-jardin apparaît alors comme le territoire des expérimentations possibles pour définir un nouveau «modèle de développement social garantissant à chacun l'amélioration des conditions d'existence $»^{37}$.

Raoul Dautry, qui s'intéresse très tôt à l'architecture, fréquente dès 1908 les penseurs qui forment le Musée social et finit en 1913 par devenir président de la plus importante commission, celle de l'habitation et de l'urbanisme. Séduit lui aussi par le concept de cité-jardin, il est influencé par les expériences menées par Howard. Aussi n'est-il pas étonnant de retrouver dans le plan d'ensemble de la cité de la Lille-La Délivrance un certain nombre d'éléments d'urbanisme caractéristiques du modèle de la "garden city » anglaise : paysage urbain marqué par la place importante qu'occupent les espaces verts - dont l'intégration dans l'environnement se fait tant au niveau des espaces publics (squares) que privés (jardins individuels) -, élégance des tracés routiers établis en courbe - semblables à ceux des parcs anglais -, choix d'édifier de petites maisons dispersées dans un cadre de verdure plutôt que des immeubles collectifs concentrés ${ }^{38}$. La constante oscillation de l'urbanisme de la cité entre ces deux pôles que sont le rural et l'urbain n'est pas sans rappeler la volonté première des promoteurs anglo-saxons du modèle de la "garden-city » de réaliser le maximum de vie rurale compatible avec la vie urbaine. À l'instar d'Ebenezer Howard, Dautry entend « faire des agents et de leurs familles des populations mi-industrielles, mi-rurales $»^{39}$, estimant que "rien n'est plus moralisateur que le contact avec la nature $»^{40}$.

Parmi les différents documents établis suite à la décision ministérielle du 12 juillet $1926^{41}$ figurent plusieurs inventaires précis des 835 logements ainsi que des édifices collectifs bâtis au sein de la cité :

« La cité [...] comprend, à gauche du chemin d'Englos :

$1^{\circ}-379$ maisons d'habitation avec annexes pour poulailler et clapier [...]. Ces

maisons comportent 824 logements [...].

$2^{\circ}$ - Une école à 4 classes avec 2 logements à 5 pièces.

$3^{\circ}$ - Un bâtiment pour bains douches avec logement.

$4^{\circ}$ - Une école maternelle à 2 classes.

$5^{\circ}$ - Une maison d'habitation pour le médecin.

$6^{\circ}$ - Une salle de réunion avec logement.

$7^{\circ}$ - Une école ménagère.

$8^{\circ}$ - Un bâtiment pour deux boutiques comprenant 2 logements à 4 pièces.

$9^{\circ}$ - Un logement de magasinier.

$10^{\circ}$ - Un bureau pour le chef de district de la Cité avec annexe pour ateliers et magasins.

$11^{\circ}$ - Un bâtiment pour le service médical avec logement.

$12^{\circ}$ - Un bâtiment provisoire pour la coopérative d'approvisionnements et un parc à combustible. Ces deux installations sont desservies par deux voies greffées sur la voie-mère d'embranchements de la gare de Lille Délivrance ${ }^{42}$. »

À cette liste, il convient d'ajouter deux maisons d'habitation pour cadres (comprenant chacune un logement) dépendant du domaine privé de la compagnie et édifiées comme logements d'agents en vertu de l'autorisation du comité en date du 16 février 1924, ainsi que le foyer des agents de train et des mécaniciens, compris dans le projet d'établissement de la gare approuvé par décision ministérielle du 30 juillet 1925. Il est intéressant de constater que les habitations des cadres ne sont pas rassemblées en un «quartier riche » mais disséminées à travers toute la cité, la compagnie refusant le 
principe d'une répartition spatiale des agents en fonction de leur statut et de leur grade : « le réseau du Nord évitait la création de deux quartiers nettement délimités, un quartier de fonctionnaires supérieurs et un quartier d'ouvriers et assourdissait ainsi, l'idée de «Lutte des classes $~_{43}$. La plupart des équipements relevant de la vie collective et sociale sont, quant à eux, concentrés dans la partie nord de la cité, autour des places de la Victoire et Beaulieu ou bien à proximité de ces dernières : c'est le cas des édifices à usage d'établissements, des bâtiments à usage de services d'assistance et d'hygiène, des installations sportives ainsi que de la salle de réunion et des boutiques. Enfin sont localisés aux confins de la cité, d'une part le service médical et le foyer des agents de train, implantés en bordure de la place Trocmet, d'autre part les ateliers et bureaux du district ainsi que la coopérative, situés le long du chemin d'Englos (fig. 1).

Figure 1. Plan d'ensemble de la cité de Lille-La Délivrance en 1926

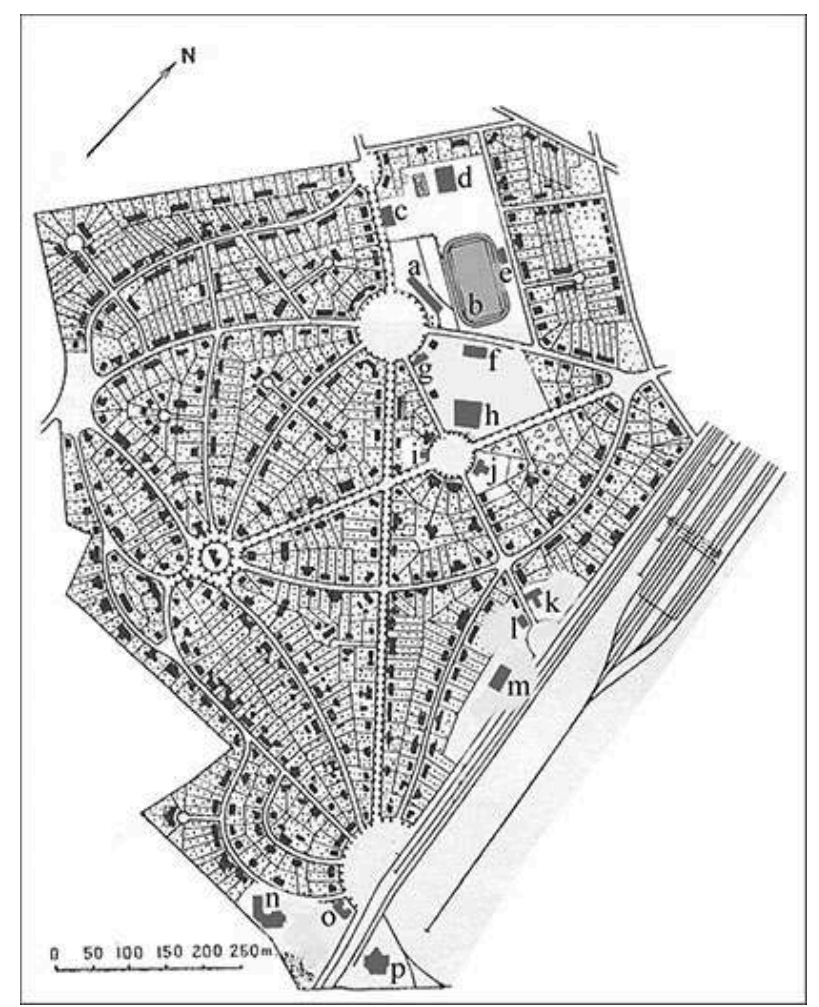

Légende :

\begin{tabular}{|l|l|}
\hline a - École primaire & i - École ménagère \\
\hline b - Terrain de sports & $j$ - Boutiques \\
\hline c - École professionnelle & k - Ateliers et bureau du district \\
\hline d - Salle de gymnastique & 1 - Logement du magasinier \\
\hline e - Bains-douches & m- Coopérative \\
\hline f-École maternelle & $n$ - Foyer des agents de trains \\
\hline
\end{tabular}




\begin{tabular}{|l|l|}
\hline $\mathrm{g}$ - Maison du docteur & o - Service médical \\
\hline $\mathrm{h}$ - Salle de réunion et des fêtes & $\mathrm{p}$ - Station d'épuration \\
\hline
\end{tabular}
verts dans le paysage urbain témoignent de l'appartenance de Dautry à l'école française d'urbanisme. L'Architecture usuelle confirme cette appartenance par le jugement d'Umbdenstock, autorité en matière d'architecture, par ailleurs architecte de la compagnie, porté sur ses réalisations : «Les cités, grandes capitales, villes secondaires ou simples villages, doivent être tenus pour des organismes vivants, des personnes collectives. Comme telles elles ont des activités et des fonctions qu'il est impossible d'envisager séparément du corps matériel et architectural où elles s'exercent. [...] L'ensemble des desiderata de la vie économique, intellectuelle et politique d'une communauté doivent s'imposer à toute édification de cité. [...] Pour [...] viser au meilleur rendement, une marche rationnelle s'indique d'elle-même. Il s'agit d'abord de dégager les divers objectifs de la vie sociale dans la ville; ensuite de chercher la coopération des formes existantes, et surtout leur coordination, c'est-à-dire déterminer celles qui doivent prendre le pas sur les autres, celles qu'il faut combattre et éliminer, celles qu'il faut faire naître. [...] C'est la tâche à laquelle a voulu se consacrer la Compagnie du Chemin de fer du Nord lorsqu'elle édifia les Cités-Jardins près des grandes villes desservies par le réseau ${ }^{44}$. »

L'édification proprement dite de la cité de La Délivrance débute en janvier 1921, soit plus d'un an après les premiers travaux de terrassement nécessaires à la gare de triage ${ }^{45}$. Désireux d'abaisser au maximum le coût unitaire de production du logement, Dautry fait de la voie ferrée "l'instrument de la rationalisation du système constructif $»^{46}$. Il écrit : «il est évident que lorsque sur un chantier des centaines de maisons s'élèvent simultanément, il est possible d'établir des voies ferrées amenant à pied d'œuvre les matériaux sans manutention et transports supplémentaires depuis leur lieu d'origine ou de fabrication jusqu'au lieu d'emploi. Pour cette mise en œuvre effectuée par quantités massives, il est possible d'utiliser un outillage puissant, en même temps qu'une organisation rationnelle du travail ${ }^{47}$." Parallèlement, "les maisons [sont] construites [...] en utilisant les agglomérés de scories dont le Réseau du Nord est producteur $»^{48}$, ces derniers étant réalisés par des équipes de cheminots dans des usines implantées pour l'occasion sur place. La réalisation du complexe ferroviaire se traduit par les «performances » suivantes : 1000000 mètres cubes de terrassements, 30000 mètres cubes de maçonnerie d'ouvrage d'art, des dizaines de kilomètres de canalisations... La cité est achevée en août 1921, soit moins d'un an après le début des travaux: pour les contemporains, "c'est une véritable ville, une ville moderne, coquette, avenante qui, en huit mois, a surgi du désert $»^{49}$.

\section{Maisons de famille et équipements collectifs}

31 Pour Raoul Dautry, la famille constitue la structure de base de la nouvelle société cheminote qu'il entend instaurer dans le cadre des cités-jardins. À ses yeux et plus généralement aux yeux de tous les réformateurs sociaux, elle garantit la stabilité morale, la solidité d'une éducation traditionnelle et efficace. Parallèlement, elle est censée, comme cela a été évoqué, favoriser une vie repliée. « Enfin, au lendemain d'une 
guerre meurtrière, elle participe pleinement d'une politique nataliste, ce qui permet à certains de dire à propos de l'action de la Compagnie qu'elle [œuvre] également pour le pays et pour la société ${ }^{50}$. En témoigne cet extrait du rapport présenté par Dautry lors des États généraux de la famille française du 12 mai 1923: "si la compagnie s'est momentanément endettée pour construire ces 40 forts que sont les cités-jardins, ces 11000 abris blindés que constituent les maisons, c'est au profit du pays. [...] Dans cette place d'armes qui couvre 800 hectares et qui est protégée par un redoutable glacis, une garnison de 38000 personnes tient solidement un des fronts de notre pays ${ }^{51}$. "

Soucieux de faire vivre l'agent dans le cadre de la vie de famille, le réseau du Nord choisit de ne faire bâtir au sein des cités que de petits logements individuels, tantôt isolés au milieu de leur jardin, tantôt réunis en groupe de deux, trois ou quatre, entourés de jardins correspondants. À Lille-La Délivrance n'est ainsi édifié aucun immeuble d'habitation collectif, à l'exception du foyer des agents de train et des mécaniciens ${ }^{52}$; quant aux 381 maisons d'habitation construites (qui comportent au total 826 logements), elles se répartissent de la manière suivante :

- 112 maisons d'habitation abritant chacune un logement (112 logements).

-157 maisons d'habitation abritant chacune deux logements (314 logements).

- 48 maisons d'habitation abritant chacune trois logements (144 logements).

- 64 maisons d'habitation abritant chacune quatre logements (256 logements).

Le nouveau monde du rail n'accepte donc qu'un type d'habitation: la petite maison individuelle. "Mère de toutes les vertus " ${ }^{53}$, elle est censée "intensifier [la] vie de famille, en y retenant le père qui n'ira plus au cabaret ; la mère, qui heureuse dans son intérieur, n'ira plus chercher du travail à l'usine voisine; les enfants qui viendront entourer leurs parents dans ce nid confortable $»^{54}$.

La composition et la disposition intérieures des logements sont le fruit d'une longue réflexion conceptuelle menée par les ingénieurs de la Compagnie du Nord qui se sont référés au modèle type des maisons à bon marché, en le modifiant, en l'interprétant et en le perfectionnant suivant leurs propres méditations. La plupart des maisons d'habitation construites comporte un étage : le rez-de-chaussée accueille les pièces de vie commune tandis que le niveau supérieur n'abrite que des chambres. Les logements y sont relativement vastes, un des objectifs poursuivis par Raoul Dautry et son équipe étant de « construire des maisons assez grandes, afin que tous n'y soient pas entassés, que l'ordre et la propreté y soient faciles, que la famille puisse s'augmenter sans crainte, et que le cheminot ne puisse plus faire cette réponse que nous avons souvent entendue dans la bouche d'ouvriers, auxquels on parlait de la nécessité pour la France d'avoir des familles nombreuses. C'est facile à dire d'avoir des enfants, mais c'est bon pour les riches qui ont de quoi les nourrir et qui peuvent les loger $\aleph^{55}$. Il est possible de distinguer deux grands types de logements : les logements du type dit salle commune, destinés aux agents subalternes, et les logements du type dit living-room, réservés aux cadres (tabl. 2). «Si l'on veut avoir une idée de la proportion dans laquelle sont répartis les différents types, sur dix logements, on peut en compter six du type salle commune à trois chambres, deux du même type à quatre et à cinq chambres et deux du type livingroom $^{56}$. $»$ Les logements du type dit salle commune se composent d'une salle commune, qui contient une cuisinière et un évier, ainsi que de trois à cinq chambres; les logements du type dit living-room, qui constituent un modèle de logement plus confortable, comptent pour leur part une salle à manger-salon, une petite cuisine séparée, une salle de bains (contrairement aux logements de « seconde classe " qui n'en 
disposent pas, ce qui explique la construction d'un établissement de bains-douches dans la cité) et de deux à cinq chambres (un petit nombre des logements du type dit living-room est conçu en vue d'héberger les cadres dirigeants de la gare de triage, chefs de section et de district ; plus grands, ils s'élèvent généralement sur trois niveaux, rezde-chaussée et deux étages). Enfin, tous les logements, quelle que soit la catégorie à laquelle ils appartiennent, sont pourvus d'un cellier faisant office de cave et de buanderie, et d'un porche « qui [...] joue de nombreux rôles : en hiver, c'est là que sont nettoyés les vêtements et les chaussures; en été, la maman s'y tient l'après-midi et travaille près de la voiture de son bébé [...] ; le père en rentrant le soir, peut s'y reposer en lisant son journal, en attendant l'heure du dîner, que toute la famille prendra ensemble, presque dans son jardin $»^{57}$. Les espaces de circulation, c'est-à-dire couloirs et escaliers, sont quant à eux réduits au strict minimum.

Tableau 2. Surface des principales pièces des logements construits à Lille-La Délivrance.

\begin{tabular}{|l|l|l|l|}
\hline \multirow{2}{*}{ Logement du type dit salle commune } & \multicolumn{2}{|l|}{ Logement du type dit living-room } \\
\hline Salle commune & \multirow{2}{*}{$18-20 \mathrm{~m}^{2}$} & Living-room & $20 \mathrm{~m}^{2}$ \\
\cline { 3 - 4 } & & Cuisine & $6 \mathrm{~m}^{2}$ \\
\hline Chambre & $11-16 \mathrm{~m}^{2}$ & Chambre & $11-16 \mathrm{~m}^{2}$ \\
\hline Cellier & $10 \mathrm{~m}^{2}$ & Cellier & $10 \mathrm{~m}^{2}$ \\
\hline Porche & $5 \mathrm{~m}^{2}$ & Porche & $5 \mathrm{~m}^{2}$ \\
\hline Salle de bains & - & Salle de bains & $5 \mathrm{~m}^{2}$ \\
\hline
\end{tabular}

Les logements construits sont vastes et bénéficient de tout le confort "moderne »: « les familles pour lesquelles on a bâti ces [...] logements, on n'a pas voulu seulement [...] leur fournir un asile; on a tenu en outre à ce qu'elles y trouvassent toutes leurs aises; on a fait en sorte de réunir pour elles les meilleures et les plus modernes conditions d'hygiène, afin d'assurer la santé physique et morale de leurs membres [...]. Bref, on a résolu de construire des maisons parfaitement adaptés aux besoins de leurs habitants ${ }^{58}$.» Ainsi toutes les habitations comprennent l'eau potable sous pression amenée à un robinet sur l'évier ainsi qu'à des bornes-fontaines (ces dernières comprenant un dispositif qui permet d'arroser les jardins et de combattre les incendies le cas échéant), un WC relié au tout-à-l'égout, l'éclairage électrique de toutes les pièces et un poêle-cuisinière installé soit dans la salle commune, soit dans la cuisine ${ }^{59}$. Chaque logement dispose en outre d'un jardin d'une superficie moyenne de cinq ares, « où l'agent, en cultivant ses légumes et quelques plantes d'agrément pour orner sa demeure, emploie ses loisirs et trouve un dérivatif au travail de la journée $»^{60}$. Il est à noter à ce propos que non seulement la pratique du jardinage est censée permettre au cheminot d'occuper son temps libre de manière "vertueuse » et " saine », mais qu'elle procure aussi un revenu supplémentaire non négligeable (l'économie réalisée étant évaluée entre 700 et 800 francs par an). Au fond de la parcelle cultivée est également bâti un poulailler-clapier. L'ensemble est clôturé par de simples fils de fer tendus sur des poteaux de ciment, peints en blanc. "Des portillons, faits de montants de bois 
géométriquement assemblés, badigeonnés de tons vifs et quelquefois coiffés d'auvents, [en] marquent l'entrée ${ }^{61}$.»

Les maisons construites dans la cité de Lille-La Délivrance doivent être spacieuses, confortables et... agréables à voir : « nous rangeons au musée des formules surannées et fausses, celle de l'inévitable séparation de l'art et de l'utilité dans la vie journalière. Nous nous rattachons au plus vieux passé français en voulant réaliser des constructions et des machines qui ne soient pas strictement utilitaires mais qui soient encore conçues et réalisées harmonieusement ${ }^{62}$. " L'édification des habitations est confiée à différents entrepreneurs ${ }^{63}$ qui s'adressent, sur la demande de la compagnie, à des architectes « de haut mérite ", à savoir, entre autres, Gustave Umbdenstock, Ernest Bertrand, Paul Piketty et le trio parisien Molinié-Nicod-Pouthier : « contrairement à l'usage qui fait de l'architecte le maître d'œuvre, on peut dire que c'est des ingénieurs ou plus exactement de M. Dautry lui-même que sont venues les idées directrices, pour être recueillies par les entrepreneurs qui ont chargé les architectes de les mettre au point. Au projet initial, solidement et largement conçu, chacun fut appelé à joindre l'apport de son invention et de son expérience. Force nous est de constater que la méthode a du bon ${ }^{64}$. » Finalement ne sont élaborés pas moins de "quatre-vingt onze types de maisons différentes " ${ }^{65}$, "d'une architecture légère, [dont la] diversité de dispositions, de détails et même de couleurs [...] chatoye agréablement ${ }^{66}$. Ainsi, sur un programme somme toute très précis, entrepreneurs et architectes, qui «ont témoigné d'un dévouement et d'une bonne volonté parfaite [...] [et se sont avérés pour la compagnie] des collaborateurs comprenant l'intérêt de l'œuvre commune ${ }^{67}$, ont su trouver des variantes heureuses et de la sorte répondre à l'attente de Raoul Dautry: bannir la monotonie, "éviter soigneusement l'uniformité ». "Cette préoccupation n'était pas seulement inspirée par des raisons esthétiques. Elle correspond encore au but philanthropique que l'on s'est proposé. Même inconsciemment, les habitants reçoivent une influence salutaire de la diversité des aspects. Ils échappent au morne ennui qui se dégage de la régularité du coron ou de la cité-caserne ${ }^{68}$. »

Figure 2. Dessin d'une maison de la cité de cheminots de Lille-La Délivrance

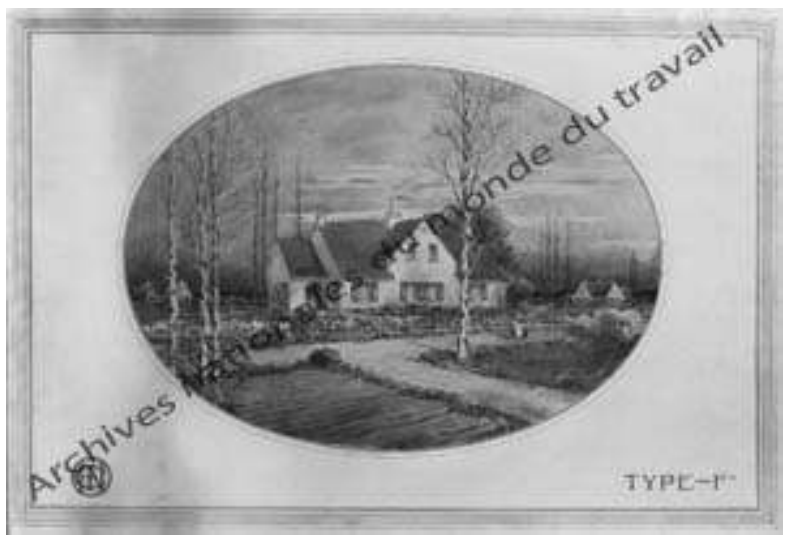


Figure 3. Photographie d'une maison de la cité de cheminots de Lille-La Délivrance. [1926]. Archives de la région Nord de la SNCF

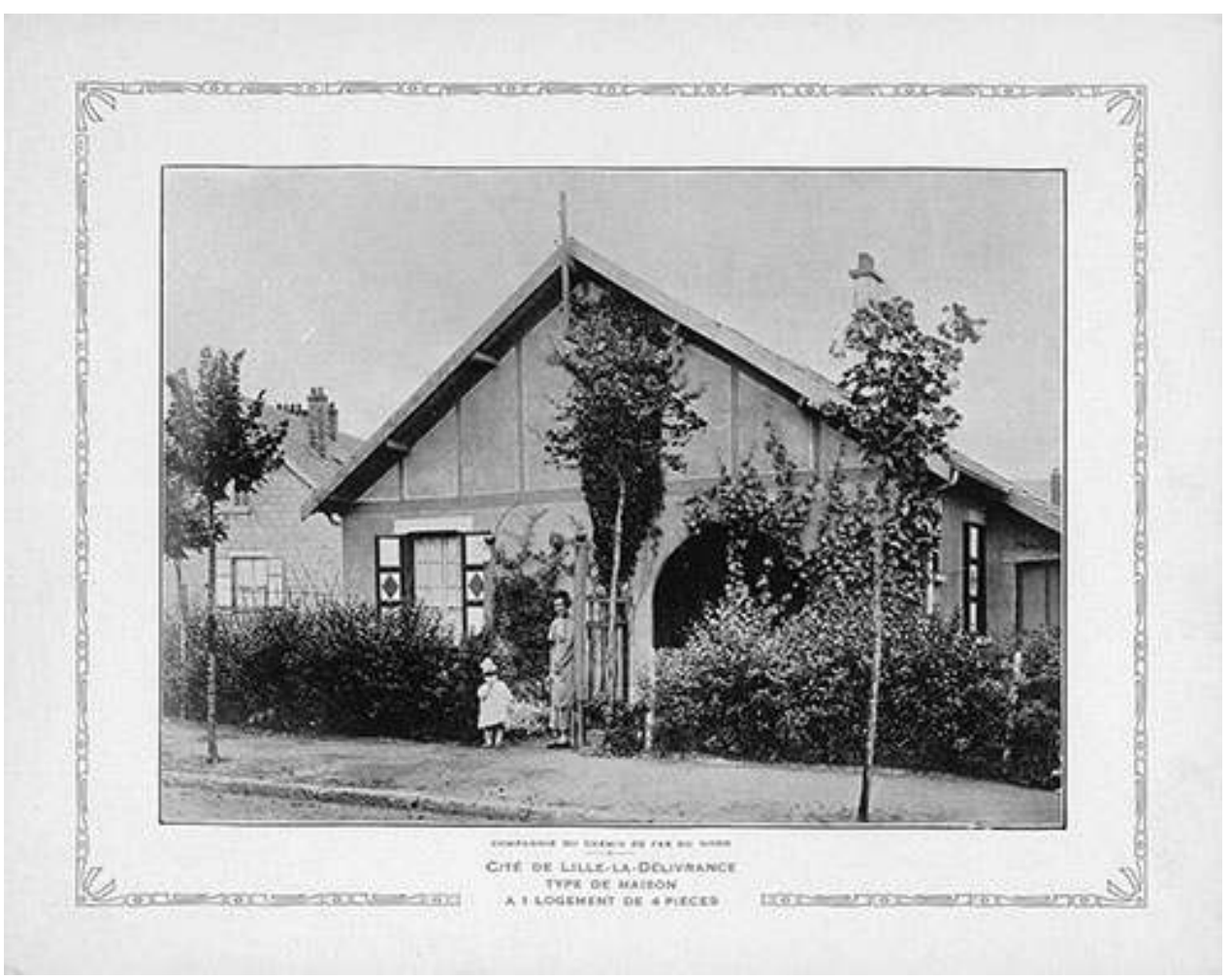

Toutes les maisons d'habitation de Lille-La Délivrance sont construites dans le style régionaliste. Déjà, en 1910, Georges Benoît-Levy, apôtre français de la cité-jardin, déclare dans un article paru dans La Gazette des beaux-arts : "pour ma part, je crois que le jour où nous créerons, en France, des cités-jardins, ce n'est pas en reniant le passé que nous trouverons le style nouveau d'architecture, mais en sachant nous en inspirer, chaque fois que ses formes seront conciliables avec la nature des matériaux qui sont à notre portée. L'architecture doit avant tout être régionale. Quelle innovation pourrait égaler le pittoresque de la maison basque, la gaieté de la ferme normande, le charme de la maison tourangelle avec ses murs honnêtement peints à la chaux et tapissés de roses grimpantes ${ }^{69}$ ? " La doctrine régionaliste, qui prône l'union entre traditions paysannes et progrès, le respect et l'adaptation des styles locaux aux exigences modernes, apparaît dans les dernières décennies du dix-neuvième siècle. S'inscrivant dans la mouvance moderniste et anti-académique, elle rallie rapidement les promoteurs de la "garden-city à la française » qui y trouvent les formes propres à concrétiser leurs rêves de foyers heureux et salubres. Aussi n'est-il pas étonnant qu'elle devienne au lendemain de la guerre le style « officiel » des cités-jardins qui se multiplient sur le territoire. Par ailleurs, parmi les grands architectes retenus par la Compagnie du Nord pour dessiner les maisons de la cité de La Délivrance (tabl. 3) ${ }^{70}$, quelques-uns se sont déjà laissés tenter par l'écriture régionaliste ; c'est le cas d'Albert Pouthier qui, associé avec les architectes parisiens Molinié et Nicod pour dessiner un certain nombre de groupes de logements de la cité, s'est illustré avant la première guerre en construisant au Touquet dans un goût régionaliste prononcé quelques belles maisons bourgeoises, comme la villa Nirvâna (1910) ${ }^{71}$. Quant à Gustave Umbdenstock (1866-1940), c'est un ardent défenseur de l'architecture régionaliste qui consacre un chapitre du cours qu'il 
professe à l'École polytechnique à «l'architecture sociale de la classe ouvrière » et y enseigne la beauté des vieilles maisons rurales ${ }^{72}$.

Les logements, qu'ils soient isolés ou groupés, sont formés de plusieurs volumes imbriqués les uns dans les autres, "harmonieusement proportionnés " aux yeux de René Chavance, auteur d'un article consacré à la cité dans la revue Art et décoration. D'amples toits, recouverts de tuiles mécaniques, les surmontent. Les murs sont rarement bâtis en briques, le plus souvent en agglomérés creux de chaux, ciment et sable, d'une épaisseur de 0,25 mètre. Afin d'assurer leur étanchéité, ils sont recouverts d'un enduit de ciment puis d'un revêtement le plus souvent blanc ou gris moucheté. Les façades, dont « beaucoup [...] de variétés [...] ont pu être obtenues dans les types, [...] et dans les détails des revêtements, les formes des fenêtres, les couleurs " ${ }^{73}$, sont percées de nombreuses ouvertures. L'entrée de chaque logement s'abrite sous un porche dont la forme varie d'un édifice à l'autre ; cependant, dans la plupart des cas, elle s'inspire de la forme traditionnelle en usage dans les anciennes demeures paysannes, avec une voûte en plein cintre soutenue par de massifs piliers en béton coupés à hauteur de soubassement par un petit mur d'appui qui, tantôt fait saillie de chaque côté de l'entrée, tantôt s'allonge d'un seul côté jusqu'au milieu du seuil. L'ensemble présente toujours un aspect très coloré : «cadre de bois et [...] contrevents [des fenêtres] sont peints de vives couleurs qui ajoutent à l'ensemble une vie et une gaieté singulières. Ces rectangles bleus, rouges, verts, jaunes, s'enlèvent en notes claires sur le fonds crème ou gris des murs. Les enduits qui protègent ceux-ci affectent des apparences diverses, simulant la pierre dans les soubassements pour se continuer jusqu'au faîte par un moucheti tyrolien très fin. Quelquefois ces crépis s'agrémentent de bandeaux ou de rayonnements de briques, soulignant de rouge vif les fenêtres et les porches. Parfois aussi [...] ils sont barrés de faux pans de bois peints en brun dont l'artifice assez vain fait penser aux maisons normandes ou alsaciennes d'exposition ${ }^{74}$.»

Tableau 3. Les architectes de la cité de Lille-La Délivrance.

\begin{tabular}{|c|c|}
\hline $\begin{array}{l}\text { Édifices particuliers ou collectifs construits à Lille-La Délivrance entre } \\
1921 \text { et } 1926\end{array}$ & Architecte \\
\hline Édifices à usage d'établissements d'enseignement : & \multirow[b]{2}{*}{ Ernest Bertranc } \\
\hline , Une école primaire à quatre classes avec deux logements de fonction & \\
\hline , Une école maternelle à deux classes & _ \\
\hline , Une école ménagère & _ \\
\hline $\begin{array}{l}\text {, Une école professionnelle (implantée dans l'ancienne salle des fêtes } \\
\text { provisoire) }\end{array}$ & - \\
\hline Édifices à usage de services d'assistance et d'hygiène : & \multirow[b]{2}{*}{ Ernest Bertrand } \\
\hline , Un bâtiment pour bains-douches avec logement de fonction & \\
\hline , Un bâtiment pour le service médical avec logement de fonction & Ernest Bertrand \\
\hline
\end{tabular}




\begin{tabular}{|c|c|}
\hline , Une maison d'habitation pour le médecin & $\begin{array}{l}\text { Gustave } \\
\text { Umbdenstock }\end{array}$ \\
\hline Autres édifices : & \\
\hline , Une salle de réunion avec logement de fonction & - \\
\hline , Un bâtiment pour deux boutiques avec deux logements de fonction & $\begin{array}{l}\text { Gustave } \\
\text { Umbdenstock }\end{array}$ \\
\hline , Un foyer pour les agents des trains & - \\
\hline $\begin{array}{l}\text {, Un bureau pour le chef de district de la cité avec annexe pour ateliers et } \\
\text { magasins }\end{array}$ & - \\
\hline $\begin{array}{l}\text {, Un bâtiment provisoire pour la coopérative d'approvisionnements et un } \\
\text { parc à combustible }\end{array}$ & - \\
\hline , Un logement de fonction pour le magasinier & - \\
\hline
\end{tabular}

Finalement, les maisons de la cité s'apparentent à des versions régionalisées des pavillons types de l'habitat à bon marché. Il est important de noter toutefois que le régionalisme des cités-jardins créées par la Compagnie du Nord n'est pas très fondamentaliste, ne respectant guère le style local ${ }^{75}$. Que ce soit à Lille-La Délivrance ou à Tergnier, on n'hésite pas en effet à mêler différents styles régionaux pour créer une variété des plus pittoresques, tant et si bien que les observateurs éprouvent bien des difficultés à rattacher les maisons érigées à un style bien défini : ainsi, alors qu'un article paru dans la revue Art et décoration explique «qu'on s'est rapproché parfois [...] des types de maisons rustiques de Picardie et des Flandres ${ }^{76}$, un journaliste de L'Écho $d u$ Nord note que «il serait très difficile d'attribuer un genre d'architecture, bien déterminé, aux maisons qui constituent la cité. Il y en a de tous types. On croit voir là un mélange de styles alsacien, suisse et hollandais. On remarque même des bâtiments qui, avec leurs crêtes de pignons en escalier, font songer à l'art espagnol $\aleph^{77}$. Un journaliste de L'Illustration pour sa part se réjouit de ce mélange de styles, écrivant que les maisons «ont [souvent] un étage aménagé sous le toit, qui, tantôt se dresse à la normande, tantôt s'abaisse à la mode basque, parfois s'encadre de redans usités en pays flamand $»^{78}$. Certains évoquent même un "curieux mais agréable style hollandosuisse $»^{79}$. Dans tous les cas, l'esthétique, particulièrement soignée, rompt avec celle du coron et des cités ouvrières traditionnelles pour s'apparenter à celle des agglomérations suburbaines et des stations balnéaires à l'usage de la bourgeoisie.

Sont conçus dans le même style que les maisons d'habitation les édifices particuliers ou collectifs de la cité, c'est-à-dire l'école primaire, l'école maternelle, l'école ménagère, la salle de réunion, les bains-douches, la maison du médecin, le service médical, les boutiques du libraire et du coiffeur et le foyer des agents de train.

41 La réception locale des édifices construits sur le territoire de la cité se révèle rapidement plutôt positive, même s'il est vrai que les matériaux et les méthodes de construction employés surprennent un peu les lommois: «les anciens Lommois ne cessaient de contempler ces constructions en parpaings gris faits de cendres et de 
ciment, au lieu des briques rouges traditionnelles. Ils ne croyaient guère à la solidité, à la durée, de ce nouveau matériau. Les anciens le disaient gravement: "Dins vingt ans, tout cha va quère in poussière" ${ }^{180}$. » Si les habitants des quartiers du Bourg et du Marais sont durant le chantier de construction quelque peu dubitatifs, la presse régionale, quant à elle, s'enthousiasme dès le départ pour l'œuvre en cours de réalisation puis, la cité construite, ne cesse de saluer la qualité de l'ensemble : La Libre Parole n'hésite pas à titrer "Une magnifique cité ouvrière " ${ }^{81}$, La Dépêche $d u$ Nord évoque "un village coquet $\aleph^{82}$. Lille-La Délivrance apparaît comme une des plus grandes cités-jardins réalisées au lendemain de la guerre par la Compagnie du Nord. Vaste, confortable, abritant un grand nombre d'habitants, elle est située en bordure de la plus grande agglomération du Nord de la France, Lille. De plus, elle occupe une position centrale au sein du réseau des cités. Tous ces éléments expliquent le fait que la direction du réseau du Nord promeuve rapidement la cité de La Délivrance, avec celles de Tergnier et de Lens, au rang de vitrine et de témoin de l'effort consenti par la compagnie dans le domaine social : " songez que vous avez un devoir moral à remplir, vous gens de la cité de La Délivrance, par votre position géographique vous êtes un centre, vous êtes un fleuron de la couronne. Si je fais un tour d'horizon, je vois des cités qui ont fait déjà de magnifiques choses. Je vois Dunkerque, la vieille Cité de Béthune, Douai, Valenciennes, Cambrai, Lens qui marchent si bien. J'en oublie probablement et je m'en excuse. Eh bien, vous, Lille, vous êtes le centre. Il faut que la Cité de Lille soit véritablement le flambeau, le phare qui éclaire tout ${ }^{83}$." De nombreux articles lui sont entièrement consacrés dans la presse nationale et spécialisée. Dans La Construction moderne par exemple sont publiées des descriptions complètes avec planches et photographies de différents édifices de la cité (maisons d'habitation, boutiques du libraire et du coiffeur, maison du docteur...). Quant aux articles qui évoquent de manière plus générale les réalisations sociales de la compagnie, Lille-La Délivrance y occupe toujours une «place de choix ». Les cités-jardins réalisées ne sont pas, aux yeux de leur créateur, que de simples «domaines" réservés aux cheminots; elles constituent aussi un modèle pertinent réformiste pouvant être appliqué à l'ensemble de la société française. À ce titre sont organisées des visites guidées et commentées de La Délivrance dont bénéficient en juillet 1922 les présidents des chambres de commerce d'Alsace-Lorraine, en août 1924 une délégation de Newcastle, en juillet 1925 les membres du congrès des médecins hygiénistes d'Amérique latine...

\section{Un village dans la ville}

\section{Une « libre commune »}

42 Construite dès 1921, la cité de Lille-La Délivrance regroupe cinq ans plus tard, en 1926, 835 logements et 3228 habitants (tabl. 4). Représentant alors un peu moins d'un cinquième de la population lommoise, elle parvient par sa taille à se hisser au rang d'un important village, voire d'un gros bourg, dépassant le chiffre fatidique des 2000 habitants qui détermine de manière administrative le seuil limite de la population rurale. Mais, sur le plan juridique, elle ne demeure rien d'autre qu'un espace privé, propriété de la Compagnie du chemin de fer du Nord, qui constitue au pire un lotissement, au mieux un des quartiers de la commune de Lomme. Cependant, dans les faits, l'agglomération cheminote apparait dès le début de son histoire comme une « libre commune », indépendante; en témoigne cet extrait d'un article paru dans L'Écho 
du Nord en 1924 : «La petite cité toute peuplée de cheminots qui s'est installée là-bas aux confins de Lomme, n'a pas fait connaître sa naissance, ni même son existence par une manifestation bruyante, avec déploiement de cortège, avec accordéons, et garde champêtre en tenue. Mais c'est une libre commune. Tout à fait libre. [...] Elle vit, pourrait-on dire, à l'écart, avec ses habitants et ses mœurs. C'est une agglomération indépendante ${ }^{84}$.» Les termes utilisés sont révélateurs: les journalistes emploient systématiquement la dénomination de "cité de Lille-La Délivrance ", présentant toujours cette dernière comme une entité distincte de la commune lommoise. Ainsi on ne parle jamais de quartier, mais de « véritable village ${ }^{85}$, de "village agricole ${ }^{86}$.

Tableau 4. Évolution de la population de la cité de Lille-La Délivrance de 1922 à 1926.

\begin{tabular}{|l|l|l|}
\hline Année & $\begin{array}{l}\text { Population de la cité } \\
\text { de Lille- } \\
\text { La Délivrance }\end{array}$ & $\begin{array}{l}\text { Population de l'ensemble des cités-jardins de la Compagnie } \\
\text { du chemin de fer du Nord }\end{array}$ \\
\hline 1922 & 2815 & 29232 \\
\hline 1923 & 2923 & 32065 \\
\hline 1924 & 3053 & 33727 \\
\hline 1925 & 3171 & 34982 \\
\hline 1926 & 3228 & 35850 \\
\hline Source : AN (CAMT), 202 AQ 1188, rapport du Comité des gestion des cités, 1927
\end{tabular}

Parallèlement, la cité, domaine cheminot par excellence, est dotée d'un conseil d'administration, "qui [est] en quelque sorte la "municipalité" de la petite ville ${ }^{87}$ : "dans chaque cité de plus de 50 logements, un conseil d'administration composé de trois fonctionnaires, nommés par le comité de gestion, et d'agents élus par leurs camarades, à raison d'un membre par 50 ménages, constitue un véritable conseil municipal. À La Délivrance, ce conseil se compose de 20 membres dont 17 agents élus par leurs pairs et 3 représentants de la compagnie ${ }^{88}$. " La présidence de cet organisme est toujours confiée par la compagnie à un des trois agents nommés. Le conseil dispose d'un budget (constitué par un prélèvement de soixante francs en moyenne par maison sur les loyers et par les recettes engendrées par l'exploitation du cinéma, de la salle des fêtes, des bains-douches) à l'aide duquel il doit assurer tous les services généraux de la cité : entretien (enlèvement des ordures ménagères, vidange des fosses, entretien des jardins publics, des rues et des installations communes, éclairage public), exploitation des différents services (service d'incendie, gérance des bains-douches, de la salle de réunion et du cinéma), organisation des fêtes, police de la cité et développement de la vie sociale (formation d'associations sportives et de loisirs, mise en place de jardins d'enfants, de cours du soir, de l'enseignement ménager,...). S'il réalise des bénéfices, il peut les utiliser à son gré pour améliorer les installations et la qualité des services consentis à la population ${ }^{89}$. Cependant, l'interprétation des historiens relève qu' « en aucune manière, [le conseil d'administration] ne peut prendre une décision qui puisse modifier l'aspect ou le règlement intérieur de la cité ${ }^{90}$; il peut seulement soumettre ses 
propositions au comité de gestion installé à Paris et qui seul détient le pouvoir de décision. Sous un semblant de démocratie qui n'a pour fonction que d'atténuer la pression patronale, la structure paternaliste, pyramidale, de la cité [...] se dévoile complètement ${ }^{91}$. Ce comité de gestion parisien se compose d'un représentant de chaque division: messieurs Schuller, inspecteur principal d'exploitation, Dullac, ingénieur principal du matériel roulant et l'incontournable Raoul Dautry. Fonctionnant comme un organe de contrôle et d'orientation du développement local des cités de la compagnie, il a tout pouvoir pour stimuler les initiatives, apaiser les discordes, trancher en dernier ressort et... augmenter ou diminuer les crédits de la cité si elle est bien ou mal tenue. En somme, conclut R. Baudoui, «l'initiative d'origine réellement locale est des plus rares. En tout état de cause, la seule stratégie dont peuvent se prévaloir les trois membres nommés du conseil d'administration (dont l'avancement est du ressort direct du supérieur hiérarchique de leur division, membre du comité de gestion) réside dans leur capacité à reprendre et amplifier les actions du développement local énoncées par ce dernier depuis la capitale $»^{92}$.

Surgie de nulle part en quelques mois, « sans avoir pris un habitant, ni même un toit, ni même un pouce de rue à aucune autre [commune] $\wedge^{93}$, pourvue de toutes les commodités et services possibles pour l'époque (ce qui est loin d'être le cas des agglomérations voisines), la « cité du rail » de La Délivrance vit dès sa construction en quasi-autarcie, n'entretenant que très peu de relations avec les autres quartiers de la ville. Aux yeux des "gens» du Bourg et du Marais, ses habitants ne sont pas des Lommois mais des cheminots dont l'univers se limite aux activités ferroviaires. Cependant, même si La Délivrance est perçue (et le sera longtemps) par beaucoup comme un " corps étranger » implanté du jour au lendemain au milieu de la commune, soucieux de préserver son indépendance, son édification marque un tournant majeur dans l'histoire de Lomme. Elle contribue ainsi fortement à l'essor démographique de l'après-guerre et, parallèlement, au renforcement du caractère ouvrier de la ville : la population lommoise passe de 11321 habitants en 1921 à 18238 habitants en 1926, soit une augmentation de $61 \%$.

\section{Vie quotidienne et contrôle social dans la cité}

Pour Raoul Dautry, selon R. Baudouï, les cités-jardins de la compagnie doivent être des espaces sociaux ayant pour objet la redéfinition des valeurs morales de la population cheminote $^{94}$. « [Selon Dautry], c'est [en effet] par le contrôle des instincts et pulsions du cheminot-ouvrier qu'il serait possible de transformer radicalement la mentalité des agents du réseau du Nord [...]. La tempérance serait reine, une vie exemplaire se déroulerait, la culture ouvrière bannie, déboucherait sur les avancées morales d'une population de cheminots procurant [...] la paix, la discipline, la confiance dans les chefs et l'amour du métier ${ }^{95}$.» Dans toutes les cités, et notamment dans celle de Lille-La Délivrance, est donc constituée une gamme très large d'équipements collectifs visant à prendre en charge d'une manière totale la vie des habitants.

Cette prise en charge doit s'exercer dès le plus jeune âge. Sont ainsi construites par la compagnie deux écoles particulièrement vastes et confortables pour l'époque. La première, située place de la Victoire et baptisée école Pasteur, est édifiée dès 1921 ; il s'agit d'une école primaire de quatre classes (deux pour les garçons et deux pour les filles). La seconde, sise rue de la Féerie et ouverte en 1924, est une école maternelle et 
comprend deux classes. Toutes deux, publiques (contrairement aux écoles des cités des compagnies minières qui sont privées), n'éprouvent guère de difficultés à recruter leurs instituteurs et institutrices, le cadre verdoyant de la cité, l'intérêt que la direction porte aux questions d'éducation (qui se traduit par la mise à disposition d'un agréable logement de fonction) et le soutien des œuvres sociales de la compagnie constituant autant d'arguments de poids pour attirer les enseignants ${ }^{96}$. Manuels et fournitures scolaires sont achetés en nombre largement suffisant tandis que sont offerts des livres destinés aux distributions de prix. Parallèlement les installations sportives de l'agglomération cheminote sont mises à la disposition des élèves : « cultiver l'esprit ne suffit pas et la Compagnie s'est préoccupée de contribuer au développement de la santé physique et de la santé morale de la jeunesse ${ }^{97}$. » Enfin la salle des fêtes et de réunion est utilisée pour organiser des séances de cinéma scolaire.

Figure 4. Photographie de l'école primaire de la cité de cheminots de Lille-La Délivrance, dite école Pasteur [1926]. Archives de la région Nord de la SNCF

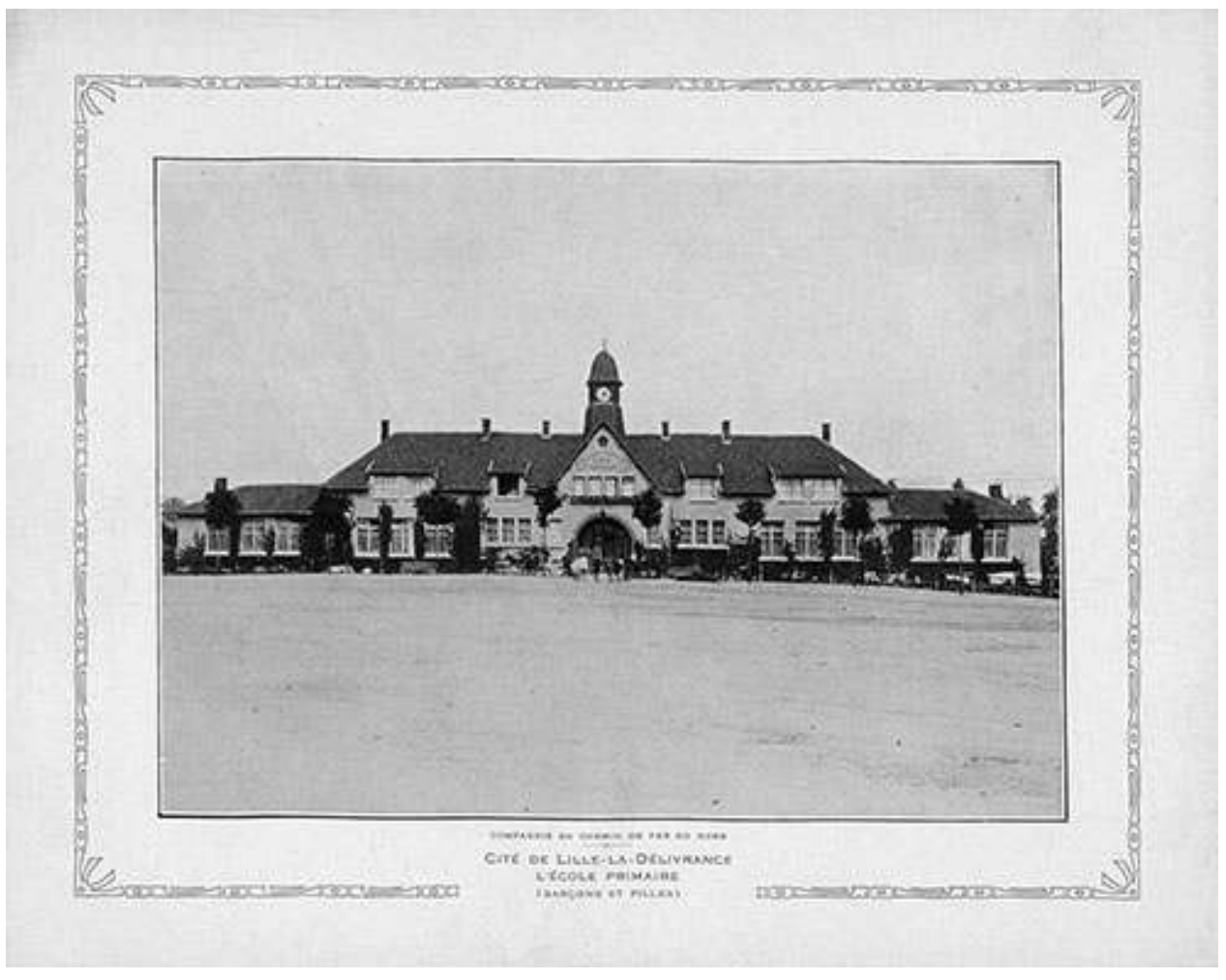


Figure 5. Photographie de la salle des fêtes et de réunion de la cité de cheminots de Lille-La Délivrance, dite salle Beaulieu [1926]. Archives de la région Nord de la SNCF

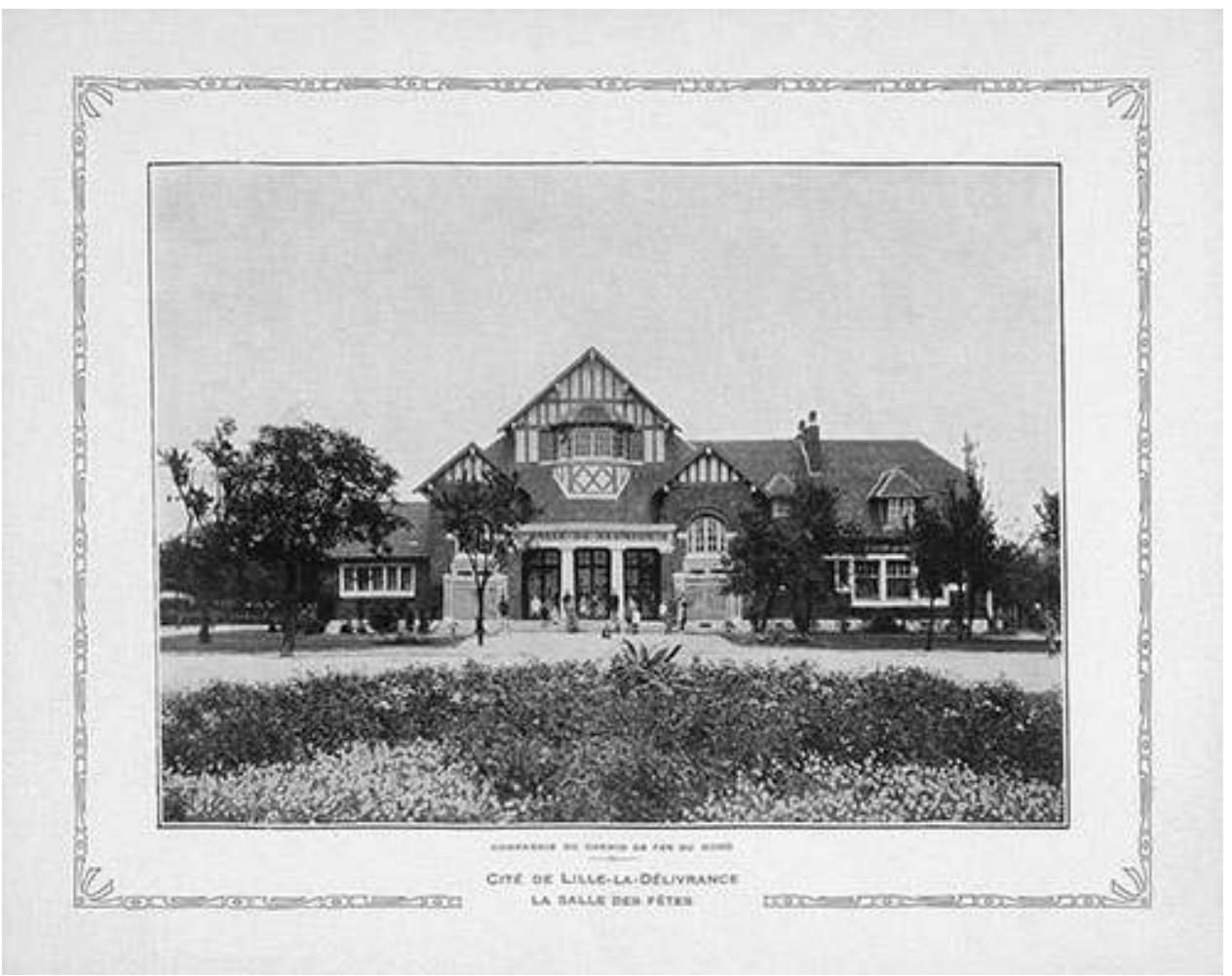

Ce dispositif est complété par la création d'un enseignement post-scolaire. Des cours d'adultes, animés par les instituteurs de la cité ainsi que par des cadres, permettent aux jeunes gens de compléter leur instruction primaire et de se préparer aux examens professionnels tandis qu'est installée dans l'ancienne salle des fêtes provisoire une école professionnelle où sont dispensés des «cours manuels » (mécanique, menuiserie, forge...). Afin de faire des filles de la cité de «futures bonnes ménagères qui sauront gérer leur ménage avec économie et en même temps créer un intérieur agréable qui retiendra le mari à la maison $»^{98}$, la compagnie aménage aussi dans une maison de quatre pièces une école ménagère: on $\mathrm{y}$ apprend « à acheter et à conserver les aliments, à préparer une nourriture à la fois saine et économique; à aménager, à décorer son intérieur, à couper et entretenir les vêtements, à réparer le linge et aussi [...] à élever les enfants $»^{99}$.

Les œuvres instituées dans la cité ne concernent pas uniquement l'enseignement ; est aussi mise en place une politique sociale dans le domaine de la santé et de l'hygiène, axée sur la prévention. Tout d'abord, Lille-La Délivrance dispose de son "docteur " attitré, nommé par la compagnie. Installé au sein d'une maison qui comprend, outre un vaste logement de fonction de neuf pièces (salon, salle à manger, cuisine, cinq chambres, une chambre de domestique), un cabinet de consultation ${ }^{100}$, il remplit les fonctions de médecin, d'éducateur, de conseiller. À ce titre, il assure la direction du service médical, structure qui sert à la fois de dispensaire et de centre d'hygiène infantile. Aidé d'une infirmière, il y reçoit les patients, donne les secours de première urgence, pratique la petite chirurgie, consulte les nourrissons. Enfin il supervise les inspections scolaires: chaque mois les enfants sont visités, pesés et est établie pour chacun d'entre eux une fiche mentionnant l'identité, l'état général, des voies respiratoires et circulatoires, l'état visuel, la dentition... ${ }^{101}$. Afin de sensibiliser la 
population des cités à un certain nombre de problèmes sanitaires, la compagnie organise ponctuellement des tournées de conférences éducatives illustrées de films ou de projections. Sont abordés au cours de ces « causeries » des sujets comme l'hygiène du corps, l'hygiène infantile, la tuberculose, le cancer ou les maladies vénériennes. Parallèlement sont apposées dans les locaux scolaires des affiches éditées par "L'hygiène par l'exemple » et le "Comité national de défense contre la tuberculose ». Quant à la question de l'alcoolisme, la direction du réseau du Nord entend résoudre le problème en interdisant tout simplement l'implantation de cafés sur le territoire de la cité $^{102}$ et la vente d'alcool dans les buvettes des salles des fêtes de cheminots : "à Tergnier, au cours d'une tournée, le comité de gestion a constaté, à la buvette de la cité, la présence d'un flacon ayant contenu de l'alcool. La vente de l'alcool étant absolument interdite dans les buvettes des cités, le comité de gestion précise qu'à la première infraction constatée, la salle des fêtes et la buvette seront définitivement fermées ${ }^{103}$. " Pour évaluer les résultats de cette politique hygiéniste, le comité de gestion exige des conseils d'administration des cités la tenue de tableaux statistiques très complets contenant toutes sortes d'indications : nombre d'habitants, d'enfants âgés de moins de quatorze ans, de naissances, de décès, de visites à domicile faites aux nourrissons, de soins et pansements effectués, d'enfants visités dans les écoles, de bains-douches donnés..., etc. Il faut noter que cette comptabilité extrêmement sophistiquée ne s'applique pas uniquement au domaine sanitaire, mais est généralisée à toutes les activités se déroulant dans les cités: enseignement, cours du soir, cours manuels et ménagers, fêtes, sociétés, rencontres sportives... Le comité parisien dispose ainsi de véritables outils d'aide à la décision, dignes de ceux réalisés aujourd'hui par les services de contrôle de gestion et d'audit ${ }^{104}$.

«L'absence de distractions saines, est seule la cause de la déchéance morale du milieu populaire. De tout temps, toutes les classes de la société ont été avides d'amusement. Les Romains réclamaient en même temps le pain et les jeux du cirque "Panem et Circenses". Ce sont ces distractions, ces amusements qu'il faut guider, et c'est un problème d'actualité beaucoup plus important aujourd'hui, depuis que la loi de huit heures laisse aux ouvriers des loisirs plus nombreux. Aussi le réseau du Nord, toujours l'un des premiers à chercher à améliorer les œuvres sociales, s'efforça-t-il à créer pour son personnel, des distractions saines et intelligentes ${ }^{105}$. » Le 6 avril 1924 est inaugurée "la maison commune ", vaste édifice qui réunit sous le même toit, outre un logement de fonction pour le concierge, une salle des fêtes (avec cinématographe), une salle de réunion pour le conseil d'administration, une buvette et une bibliothèque. Cette dernière, constituée essentiellement à l'aide de dons, compte en 1926 quelques 576 ouvrages (ce qui se révèle peu important comparé aux fonds des bibliothèques des cités de Tergnier et de Lens, la première conservant 3820 ouvrages, la seconde 1861 ). Un tel équipement socioculturel doit contribuer à « rendre le séjour de la cité le plus attrayant possible [afin] d'éviter que les habitants aillent, chaque dimanche, chercher à Lille, les distractions qu'ils ne peuvent trouver dans leur cité ${ }^{106}$. Parallèlement, des installations sportives permettent de «faire naître et [d'] entretenir chez [les cheminots] le goût de la culture physique ${ }^{107}$ : stade, terrain de jeux, salle de gymnastique et même tennis. Tous ces moyens (qui sont vraiment en terme d'infrastructure très importants pour l'époque) sont mis à la disposition des nombreuses sociétés de cheminots.

50 "Lille-La Délivrance est une des cités où les sociétés ont pris un grand essor ${ }^{108}$." Suscitées sur initiatives locales, elles sont rapidement appropriées et contrôlées par le 
comité de gestion qui voit en elles un parfait outil d'encadrement de la vie sociale dans les cités. Régulièrement les réunions du comité de gestion abordent par cité la question de l'aide financière à répartir. Cette dernière s'apparente à une ouverture faite aux associations remplissant sur le terrain les engagements et les objectifs sociaux qui leur ont été assignés. Sorte de prime ou rançon d'un succès, elle est octroyée sur la base d'un ensemble de critères disparates qui recouvrent aussi bien l'ancienneté de la société, les difficultés ayant trait à sa création que le nombre d'actifs ${ }^{109}$. Dans tous les cas, les sociétés de la cité de Lille-La Délivrance, tout comme celles des autres citésjardins de la compagnie, sont largement « utilisées » par le comité de gestion parisien pour animer la vie quotidienne de l'agglomération. Sont ainsi organisées nombre de fêtes et de manifestations sportives ou culturelles : en 1926 ont lieu dans la cité pas moins de quatorze concerts et représentations théâtrales, quatre fêtes données par la Société de sport et d'athlétisme. Leur programmation est le fruit d'un savant dosage qui vise à alterner judicieusement activités sportives, récréatives et pédagogiques. L'objectif poursuivi est double : retenir les cheminots et leur famille sur le territoire de la cité d'une part, développer un esprit de solidarité entre les agents d'autre part.

51 Lors de la construction de la cité, une coopérative provisoire est installée. Elle reçoit les fournitures par train: sur une voie ferrée desserte, un quai est aménagé pour permettre les chargements et les déchargements. L'objectif d'une telle initiative est de permettre aux ménagères l'achat habituel des provisions sans avoir à sortir des limites de l'agglomération cheminote. Parallèlement, afin de limiter au maximum «les intrusions extérieures ", « les seuls commerçants dont la présence [est] admise dans la Cité [sont] le libraire et le coiffeur $\aleph^{110}$. La Compagnie du Nord entend donc répondre à tous les besoins de ses agents, s'immiscer dans tous les aspects de leur vie quotidienne : enseignement, santé, loisirs, approvisionnement. Il reste cependant un domaine dans lequel elle ne souhaite pas intervenir: le culte. Raoul Dautry estime en effet que la nouvelle société cheminote doit échapper aux divisions traditionnelles de la société civile. Aussi n'hésite-t-il pas à déclarer, lors de la cérémonie d'inauguration de la salle des fêtes et de réunion de la cité : «l'idéal religieux, est, pour beaucoup, la plus radieuse espérance et la plus pure consolation; l'idéal politique procure, à ceux qui le poursuivent, l'utilisation de leur activité, l'emploi de leur combativité et, somme toute, de viriles satisfactions. Mais je souhaite que l'un et l'autre restent au fond du cœur des cheminots tant qu'ils seront dans la cité et ne s'expriment qu'au dehors, car ils n'unissent pas souvent, et fréquemment ils divisent. Or tout ce qui peut diviser dans les cités est pour moi à écarter. En ami de tous, je ne veux considérer que les choses qui vous unissent ${ }^{111}$." Ces considérations expliquent donc le fait que «la compagnie n'[installe] [...] ni écoles confessionnelles, ni églises, ni presbytères [et que] les habitants des cités [aient] recours aux établissements des villages voisins [...]. Toutefois cinq cités ont des églises ou des chapelles en bois : Lens, Saint-Pol-sur-Mer, Aulnoye, Buire, Hirson, Busigny. Mais, dans ce cas, l'achat et l'installation sont du domaine exclusif de l'évêché » ${ }^{112}$.

\section{Conclusion}

Que reste-t-il aujourd'hui du monde clos de la cité de Lille-La Délivrance? Nombre d'édifices ont été détruits suite au bombardement d'avril 1944, qui constitue le drame le plus effroyable de l'histoire de la cité. Dans la nuit du 9 au 10 avril, s'abat, en vingt- 
cinq minutes, un véritable déluge de feu sur l'ensemble ferroviaire. Le bilan est particulièrement lourd: 106 tués, plus de 300 blessés tandis que, sur les 850 logements que compte l'agglomération, $1 / 5^{\mathrm{e}}$ est complètement détruit, $3 / 5^{\mathrm{e}}$ sont fortement endommagés et $1 / 5^{\mathrm{e}}$ réparable dans un délai plus ou moins bref ${ }^{113}$. Quant aux équipements collectifs construits entre 1921 et 1924, nombre doivent être abattus : l'école Pasteur, les bains-douches, le service médical..., etc. Après le conflit, la cité voit le désengagement progressif de la SNCF, héritière de la Compagnie du Nord depuis 1937 : rétrocession à la commune de bon nombre d'édifices collectifs, vente de maisons à des particuliers. L'habitat de la cité évolue lui aussi profondément. La reconstruction ne tient plus compte des principes si chers à Dautry: les nouvelles habitations sont bâties en brique, par souci d'économie, et ressemblent désormais à toutes celles bâties dans la région lilloise tandis que les jardins sont de moins en moins entretenus et les constructions provisoires se multiplient. Malgré tous ces éléments, la cité de La Délivrance conserve encore un nombre non négligeable d'édifices construits dans les années vingt, à la valeur architecturale remarquable.

\section{NOTES}

1. Archives Nationales (Centre des archives du monde du travail, Roubaix, désormais: AN (CAMT), 48 AQ 574, rapport sur l'exercice 1918 présenté par le conseil d'administration de la compagnie lors de l'assemblée générale des actionnaires, 1919, p. 11.

2. Ibid., p. 11-12.

3. » La gare de Lille-La Délivrance ", Le Monde illustré, 1923, tome 10, p. 29.

4. Direction régionale de Lille de la SNCF, archives du service des bâtiments, note de M. Caille, chef de bureau adjoint du service EX, 1955.

5. Il est à noter toutefois qu'une petite partie du complexe ferroviaire de La Délivrance se situe sur le territoire de la commune de Sequedin.

6. Direction régionale de Lille de la SNCF, archives du service des bâtiments, note citée, 1955.

7. Rémi Baudoui, - Rémi Baudoui, Raoul Dautry. 1880-1951. Le technocrate de la République, Paris, Éditions Balland, 1992, p. 62-64.

8. Pierre Outteryck, Mémoire cheminote en Nord-Pas-de-Calais. Cheminots et Chemins de Fer du Nord. 1938-1948, Lille, CER SNCF de Lille, 1995, p. 16.

9. Jules Brenne, «Aspects historiques et régionaux de la construction d'une grande gare de triage : la Délivrance. 1920 ", Bulletins de la Commission historique du département du Nord, 1970, tome 38 , p. 57.

10. Maxime Ducrocq, «Les habitations ouvrières dans la région du Nord », Le Monde illustré, 1923, tome 10 , p. 27.

11. AN (CAMT), 48 AQ 3399, courrier des administrateurs de la Compagnie du chemin de fer du Nord au ministre des Travaux publics et des Transports, 25 mars 1919.

12. Pierre Outteryck, op. cit., p. 17.

13. «Réalisations sociales. les cités-jardins sur le Nord ", L'Écho de Paris, 18 août 1924.

14. Les informations relatives à Raoul Dautry proviennent des ouvrages et articles suivants :

- Rémi Baudoui, Raoul Dautry. 1880-1951. Le technocrate de la République, Paris, Éditions Balland, 1992. 
- Rémi Baudoui, «La cité-jardin de Tergnier de la Compagnie du Nord, 1921-1950. Éléments d'analyse d'un modèle de société cheminote " in «Les chemins de fer, l'espace et la société en France ", Actes du $1^{\text {er }}$ colloque de l'AHICF (Paris, mai 1988), Revue d'histoire des chemins de fer hors série, 1, 1989, p. 264-277.

- Rémi Baudoui, «La cité-jardin française entre mythes et réalités » in Cités, cités-jardins : une histoire européenne, Talence, Editions de la maison des sciences de l'homme d'Aquitaine, 1996, p. 87-100.

- Bernard Ghienne, «La cité des cheminots » in «Lens, la gare, le dépôt, la cité des cheminots », Les Dossiers de Gauberia, n 5, 1996, p. 67-141.

15. Rémi Baudoui, op. cit., 1992, p. 33.

16. Rémi Baudoui, art. cité, 1989, p. 267.

17. Ibid.

18. Raoul Dautry, «La rationalisation de la cité », L'Illustration, n 4491, 1929.

19. Patrick Favardin, «Tergnier, la cité-jardin des cheminots ", Monuments historiques, $n^{\circ} 6 / 1978$, p. 34 .

20. Maurice Boisseau, Les CEuvres d'amélioration sociale dans la Compagnie des chemins de fer du Nord. Thèse pour le doctorat (Sciences politiques et économiques) présentée et soutenue le 6 décembre 1924, à 16 heures, Paris, Imprimerie librairie militaire universelle L. Fournier, 1924, p. 38.

21. » Les cités-jardins des Chemins de fer du Nord ", La Construction moderne, 14 octobre 1923, p. 13.

22. » Une nouvelle cité ouvrière à Lille-Délivrance ", L'Écho du Nord, 4 février 1922.

23. René Chavance, «Les cités jardins de la Compagnie du Nord », Art et décoration, octobre 1922, p. 118.

24. Maurice Boisseau, thèse citée, p. 37.

25. René Chavance, art. cité, p. 118.

26. Signalons aussi la place de la Victoire sur laquelle est construit en 1928 un kiosque à musique.

27. Antony Goissaud, «L'œuvre de la Compagnie du Nord. Cité-jardin de Lille-La Délivrance », La Construction moderne, 20 mars 1927, p. 287.

28. Il est à noter qu'est aménagé dès 1926 sur la partie centrale de la place François- Marcq un terrain de jeu de balle. Des vestiaires sont installés à proximité.

29. AN (CAMT), 202 AQ 1188, rapport du Comité des gestion des cités, 1927, p. 62.

30. Raoul Dautry, «Les cités-jardins du Chemin de fer du Nord », Revue d'hygiène, 1922, p. 13.

31. Paul Calfas, "Les cités-jardins de la Compagnie des chemins de fer du Nord", Le Génie civil. Revue générale hebdomadaire des industries françaises et étrangères, tome 84, (1924), nº 2168, p. 199.

32. Soulignons à ce propos le fait que la cité ne compte aucun immeuble de logement collectif, à l'exception du foyer des agents de train et des mécaniciens.

33. «La visite des représentants des Chambres de Commerce d'Alsace et de Lorraine. La gare de Lille-Délivrance et les Mines de Lens », Le Nord maritime, 29 juillet 1922.

34. À titre de comparaison, il est intéressant de noter qu'en 1926 la cité-jardin du Rondeau, située près de Grenoble et édifiée quelques années plus tôt, compte 1032 habitants, logés dans 200 logements répartis sur un terrain d'une superficie d'un peu moins de 9 hectares, soit une densité de 114 habitants par hectare (cf. Thierry Maillot ; Yann Seroz ; Jean-Marc Vidal, Un village dans la ville. La cité-jardins Paul Mistral. Grenoble. 1925-1960, Vénissieux, Éditions Paroles d'Aube, 1996). Parallèlement, lorsqu'en 1924 le département de la Seine souhaite mettre en place un grand programme de construction de logements sociaux, il lance un concours en vue de créer une cité de 80000 habitants sur 700 hectares de terrains, ce qui sous-entend à nouveau une densité de 114 habitants par hectare (cf. Rémi Baudoui, art. cité, 1996, p. 97).

35. Leonardo Benevolo, Histoire de l'architecture moderne. Avant-garde et mouvement moderne (1890-1930), trad. fr., Paris, Dunod, 1979, p. 108. 
36. Idées de cité-jardins. L'exemplarité de Suresnes, Suresnes, édité par la Ville de Suresnes, 1998, p. 11.

37. Rémi Baudoui, art. cité, 1996, p. 89.

38. Il est à noter qu'Howard ne s'occupe pas d'architecture et ne précise ni le tracé de la ville, ni le style des édifices; ce sont les architectes qui travaillent avec lui, à savoir Raymond Unwin et Barry Parker, qui conçoivent les plans de Letchworth.

39. AN (CAMT), 202 AQ 1189, rapport présenté par Raoul Dautry aux États généraux de la Famille française, le 12 mai 1923.

40. AN (CAMT), 202 AQ 1189, rapport présenté par Raoul Dautry au Congrès de l'habitation, le 9 décembre 1922.

41. La décision ministérielle du 12 juillet 1926 approuve à titre de régularisation comme suite et complément des travaux déclarés d'utilité publique pour la construction de la ligne d'Haubourdin à Saint-André, le projet d'établissement de la cité de Lille-La Délivrance.

42. AN (CAMT), 202 AQ 1191, rapport estimatif accompagnant la décision ministérielle du 12 juillet 1926 citée.

43. Maurice Boisseau, thèse citée, p. 36 .

44. Gustave Umbdenstock, "Cités-jardins de la Compagnie du Chemin de fer du Nord", L'Architecture usuelle, 1922, volume 3, p. 189, 191.

45. Petit rappel en matière de chronologie : décembre 1920 : début des travaux de construction de la gare de triage; janvier 1921 : début des travaux de construction de la cité ; août 1921 : fin des travaux de construction de la cité ; $1^{\mathrm{er}}$ octobre 1921 : mise en service de la gare de triage.

46. Rémi Baudoui, op. cit., 1992, p. 63.

47. Raoul Dautry, art. cité, 1929.

48. Raoul Dautry, «Les installations du personnel », L'Ingénieur-constructeur, n 148, 1923, p. 19.

49. «La visite des représentants des Chambres de Commerce d'Alsace et de Lorraine. La gare de Lille-Délivrance et les Mines de Lens ", Le Nord maritime, 29 juillet 1922.

50. Patrick Favardin, art. cité, p. 34.

51. AN (CAMT), 202 AQ 1189, rapport présenté par Raoul Dautry aux États généraux de la famille française, le 12 mai 1923, p. 16.

52. Exception faite de la cité construite au Bourget.

53. Patrick Favardin, art. cité, p. 33.

54. Maurice Boisseau, thèse citée, p. 22.

55. Ibid., p. 22-23.

56. René Chavance, art. cité, p. 120.

57. Maurice Boisseau, thèse citée, p. 23.

58. René Chavance, art. cité, p. 113.

59. L'alimentation en eau potable des maisons de la cité est assurée par un réseau de canalisations prenant son origine à deux réservoirs de 200 mètres cubes alimentés par l'eau de la Ville de Lomme. Un second réseau de canalisations dirige les produits des WC, les eaux pluviales et ménagères vers une station d'épuration située dans les emprises de la gare.

60. AN (CAMT), 48 AQ 3399, rapport établi par les services de la Compagnie du Nord intitulé « L'effort social du Réseau du Nord dans le département du Nord », 1933.

61. René Chavance, art. cité, p. 119.

62. AN (CAMT), 202 AQ 1189, rapport présenté par Raoul Dautry au Congrès de l'habitation, le 9 décembre 1922.

63. Les entrepreneurs auxquels a fait appel la Compagnie du Nord sont les Établissements Féron frères, la Société D. et N. Rolland frères, la Société de construction et de reconstruction, l'Entreprise générale Pelle-Goudard et Cie et la Société des bâtiments du Nord et de l'Est. Cette liste est cependant très certainement incomplète.

64. René Chavance, art. cité, p. 112. 
65. Antony Goissaud, art. cité, p. 288.

66. « Une magnifique cité ouvrière », La Libre Parole, 22 juin 1921.

67. Raoul Dautry, art. cité, 1923, p. 20.

68. René Chavance, art. cité, p. 122.

69. Cité par Jean-Claude Vigato, «La cité-jardin et l'architecture régionaliste » in Cités, citésjardins: une histoire européenne, Talence, Éditions de la maison des sciences de l'homme d'Aquitaine, 1996, p. 116.

70. Il est intéressant de noter à ce propos que tous les architectes employés pour le chantier de construction de la cité de La Délivrance se révèlent être vite des partenaires privilégiés de la compagnie, tous participant aussi à la conception d'édifices construits dans d'autres cités du réseau du Nord, comme celles de Tergnier ou de Lens.

71. Richard Klein, La Côte d'Opale des années trente. Le Touquet Paris-Plage, Paris, Éditions Norma, 1994, p. 77-78.

72. Jean-Claude Vigato, art. cité, p. 118.

73. Maurice Boisseau, thèse citée, p. 27.

74. René Chavance, art. cité, p. 126.

75. Jean-Claude Vigato, art. cité, p. 118.

76. René Chavance, art. cité, p. 127.

77. » Lille-Délivrance. La grande gare de triage, progressivement, prend l'aspect d'une ville nouvelle ", L'Écho du Nord, 3 septembre 1921.

78. " Les cités-jardins des cheminots du Nord », L'Illustration, 13 octobre 1923.

79. » Une libre commune de 4500 âmes aux portes de Lille », L'Écho du Nord, 29 septembre 1924.

80. Jules Brenne, art. cité, p. 62.

81. " Une magnifique cité ouvrière », art. cité.

82. " Lille. Les délégués des Chambres de commerce d'Alsace-Lorraine, à Lille-Délivrance ", La Dépêche du Nord, 29 juillet 1922.

83. AN (CAMT), 202 AQ 1192, discours prononcé par Monsieur Javary, directeur de l'exploitation, lors de l'inauguration de la salle des fêtes et de réunion de la cité de Lille-La Délivrance, 5 octobre 1924.

84. » Une libre commune aux portes de Lille », L'Écho du Nord, 29 septembre 1924.

85. » Lille. L'extension du trafic ferroviaire. La gare de Lille-Délivrance », Le Télégramme du Nord, 13 janvier 1921.

86. " Sequedin. La gare de Lille-Délivrance s’édifie », ibid., 31 mars 1921.

87. " Une magnifique cité ouvrière ", art. cité.

88. AN (CAMT), 202 AQ 1189, rapport présenté par Raoul Dautry au congrès de l'habitation, le 9 décembre 1922.

89. AN (CAMT), 202 AQ 1189, procès-verbaux des réunions du comité de gestion des cités du 3 juin 1920 et du 8 juillet 1920 .

90. Nous ne sommes pas parvenus à retrouver un exemplaire de ce règlement.

91. Patrick Favardin, art. cité, p. 34.

92. Rémi Baudoui, art. cité, 1989, p. 272.

93. " Une libre commune aux portes de Lille », art. cité.

94. Rémi Baudoui, art. cité, 1989, p. 270.

95. Rémi Baudoui, op. cit., 1992, p. 64.

96. Bernard Ghienne, art. cité, p. 127.

97. Raoul Dautry, art. cité, 1922, p. 18.

98. AN (CAMT), 202 AQ 1188, compte rendu du conseil d'administration de la cité de Lille-La Délivrance sur les écoles ménagères, 1925.

99. Maurice Boisseau, thèse citée. 
100. Pour plus d'informations concernant la maison du docteur, voir l'article cité d'Antony Goissaud qui en fournit une description très précise.

101. AN (CAMT), 202 AQ 1189, procès-verbal de la réunion du comité de gestion des cités du 21 février 1922.

102. Cette décision ne répond pas uniquement à un souci d'ordre hygiéniste. Il s'agit aussi pour la compagnie d'une manière d'éviter les risques d'agitation et de contrer la menace que représentent les syndicats, ces derniers établissant traditionnellement leur siège social dans un débit de boissons où ils rassemblent adhérents et sympathisants.

103. AN (CAMT), 202 AQ 1189, procès-verbal de la réunion du comité de gestion des cités du 6 mars 1928.

104. Il est possible de retrouver tous ces tableaux statistiques dans : AN (CAMT), 202 AQ 1188, rapport du comité de gestion des cités, 1927.

105. Maurice Boisseau, thèse citée, p. 78.

106. AN (CAMT), 202 AQ 1189, procès-verbal de la réunion du comité de gestion des cités du 31 octobre 1922.

107. AN (CAMT), 202 AQ 1188, rapport du comité de gestion des cités, 1927, p. 193.

108. Ibid., p. 63.

109. Rémi Baudoui, art. cité, 1989, p. 273.

110. AN (CAMT), 48 AQ 3399, rapport établi par les services de la Compagnie du Nord intitulé "L'effort social du Réseau du Nord dans le département du Nord », 1933.

111. Cité par Rémi Baudoui, art. cité, 1989, p. 268.

112. AN (CAMT), 202 AQ 1191, note du secrétariat général, 25 novembre 1929.

113. Direction régionale de Lille de la SNCF, archives du service des bâtiments, compte rendu du bombardement de la cité de Lille-La Délivrance dans la nuit du 9 au 10 avril 1944.

\section{AUTEUR}

\section{ARNAUD GABORIAU}

Titulaire d'un certificat d'études approfondies en architecture, École d'architecture de Lille 